\title{
An analytic study of the off-diagonal mass generation for Yang-Mills theories in the maximal Abelian gauge
}

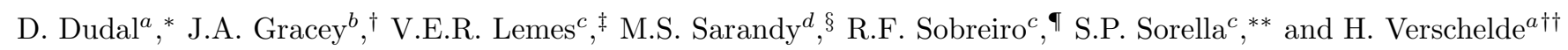

a Ghent University

Department of Mathematical Physics and Astronomy

Krijgslaan 281-S9

B-9000 Gent, Belgium

b Theoretical Physics Division

Department of Mathematical Sciences

University of Liverpool

P.O. Box 147, Liverpool, L69 3BX, United Kingdom

c UERJ - Universidade do Estado do Rio de Janeiro

Rua São Francisco Xavier 524, 20550-013 Maracanã

Rio de Janeiro, Brazil

d Chemical Physics Theory Group, Department of Chemistry,

University of Toronto, 80 St. George Street, Toronto, Ontario, M5S 3H6, Canada

\begin{abstract}
We investigate a dynamical mass generation mechanism for the off-diagonal gluons and ghosts in $S U(N)$ Yang-Mills theories, quantized in the maximal Abelian gauge. Such a mass can be seen as evidence for the Abelian dominance in that gauge. It originates from the condensation of a mixed gluon-ghost operator of mass dimension two, which lowers the vacuum energy. We construct an effective potential for this operator by a combined use of the local composite operators technique with the algebraic renormalization and we discuss the gauge parameter independence of the results. We also show that it is possible to connect the vacuum energy, due to the mass dimension two condensate discussed here, with the non-trivial vacuum energy originating from the condensate $\left\langle A_{\mu}^{2}\right\rangle$, which has attracted much attention in the Landau gauge.
\end{abstract}

PACS numbers: 11.10.Gh,12.38.Lg

\section{INTRODUCTION.}

A widely accepted mechanism to explain color confinement in $S U(N)$ Yang-Mills theories is based on the dual superconductivity picture [1, 2], according to which the low energy regime of QCD should be described by an effective Abelian theory in the presence of magnetic monopoles. These monopoles should condense, giving rise to a string formation à la Abrikosov-Nielsen-Olesen. As a result, chromoelectric charges are confined. This mechanism has received many confirmations from the lattice community in the so-called Abelian gauges, which are useful in order to isolate the effective relevant degrees of freedom at low energy.

According to the concept of Abelian dominance, the low energy region of QCD can be expressed solely in terms of Abelian degrees of freedom [3]. Lattice confirmations of the Abelian dominance can be found in [4, 5]. A particularly interesting Abelian gauge is the maximal Abelian gauge (MAG), introduced in 2, 6, 7]. Roughly speaking, the MAG is obtained by minimizing the square of the norm of the fields corresponding to off-diagonal gluons, i.e. the gluons associated with the $N(N-1)$ off-diagonal generators of $S U(N)$. Doing so, there is a residual $U(1)^{N-1}$ Abelian gauge freedom corresponding to the Cartan subgroup of $S U(N)$. The renormalizability in the continuum of this gauge was proven in [8, 9], at the cost of introducing a quartic ghost interaction.

\footnotetext{
${ }^{*}$ Research Assistant of the Fund For Scientific Research-Flanders (Belgium); Electronic address: david.dudal@ugent.be

${ }^{\dagger}$ Electronic address: jag@amtp.liv.ac.uk

${ }^{\ddagger}$ Electronic address: vitor@dft.if.uerj.br

§Electronic address: msarandy@chem.utoronto.ca

IElectronic address: sobreiro@uerj.br

**Electronic address: sorella@uerj.br

$\dagger^{\dagger}$ Electronic address: henri.verschelde@ugent.be
} 
To our knowledge, there is no analytic proof of the Abelian dominance. Nevertheless, an argument that can be interpreted as evidence of it, is the fact that the off-diagonal gluons would attain a dynamical mass. At energies below the scale set by this mass, the off-diagonal gluons should decouple, and in this way one should end up with an Abelian theory at low energies. A lattice study of such an off-diagonal gluon mass reported a value of approximately $1.2 \mathrm{GeV}$ [10]. More recently, the off-diagonal gluon propagator was investigated numerically in [11], reporting a similar result.

There have been several efforts to give an analytic description of the mechanism responsible for the dynamical generation of the off-diagonal gluon mass. In [12, 13], a certain ghost condensate was used to construct an effective, off-diagonal mass. However, in [14] it was shown that the obtained mass was a tachyonic one, a fact confirmed later in [15]. Another condensation, namely that of the mixed gluon-ghost operator $\left(\frac{1}{2} A_{\mu}^{a} A^{\mu a}+\alpha \bar{c}^{a} c^{a}\right)$ [54], that could be responsible for the off-diagonal mass, was proposed in [16]. That this operator should condense can be expected on the basis of a close analogy existing between the MAG and the renormalizable nonlinear Curci-Ferrari gauge [17, 18]. In fact, it turns out that the mixed gluon-ghost operator can be introduced also in the Curci-Ferrari gauge. A detailed analysis of its condensation and of the ensuing dynamical mass generation can be found in [19, 20].

The aim of this paper is to investigate explicitly if the mass dimension two operator $\left(\frac{1}{2} A_{\mu}^{a} A^{\mu a}+\alpha \bar{c}^{a} c^{a}\right)$ condenses, so that a dynamical off-diagonal mass is generated in the MAG. The pathway we intend to follow is based on previous research in this direction in other gauges. In 21], the local composite operator (LCO) technique was used to construct a renormalizable effective potential for the operator $A_{\mu}^{A} A^{\mu A}$ in the Landau gauge. As a consequence of $\left\langle A_{\mu}^{A} A^{\mu A}\right\rangle \neq 0$, the gauge bosons acquired a mass [21]. The fact that gluons in the Landau gauge become massive has received confirmations from lattice simulations, see for example 22]. Recently, the dynamical mass generation in the Landau gauge has been investigated within the Schwinger-Dyson formalism in 23,24$]$. The condensate $\left\langle A_{\mu}^{A} A^{\mu A}\right\rangle$ has attracted attention from theoretical [25, 26] as well as from the lattice side [27, 28, 29]. It was shown by means of the algebraic renormalization technique [30] that the LCO formalism for the condensate $\left\langle A_{\mu}^{A} A^{\mu A}\right\rangle$ is renormalizable to all orders of perturbation theory 31]. The same formalism was successfully employed to study the condensation of $\left(\frac{1}{2} A_{\mu}^{A} A^{\mu A}+\alpha \bar{c}^{A} c^{A}\right)$ in the Curci-Ferrari gauge [19, 20]. We would like to note that the Landau gauge corresponds to $\alpha=0$. Later on, the condensation of $A_{\mu}^{A} A^{\mu A}$ was confirmed in the linear covariant gauges [32, 33], which also possess the Landau gauge as a special case. It was proven formally that the vacuum energy does not depend on the gauge parameter. However, in practice, a problem occurred due to the mixing of different orders of perturbation theory, when solving the gap equation for the condensate. Nevertheless, we have been able to present a way to overcome this problem [33]. As a result, it turns out that the non-trivial vacuum energy due to the condensate $\left\langle A_{\mu}^{A} A^{\mu A}\right\rangle$ in the Landau gauge coincides with the non-trivial vacuum energy due to the appropriate mass dimension two condensate in the linear covariant gauges, $\left\langle A_{\mu}^{A} A^{\mu A}\right\rangle$, and the Curci-Ferrari gauge, $\left\langle\frac{1}{2} A_{\mu}^{A} A^{\mu A}+\alpha \bar{c}^{A} c^{A}\right\rangle$, since these two classes of gauges both have the Landau gauge, $\alpha=0$, as a limiting case.

We would also like to underline that the concept of a gluon mass has already been widely used in a more phenomenological context since long ago, see e.g. 34]. More recently, a gluon mass of the order of a few hundred MeV has been proven to be very useful in describing the radiative decay of heavy quarkonia systems [35] as well as to derive estimates of the glueball spectrum [36].

To make this paper self-contained, we will explain all necessary steps in the case of the MAG, and refer to the previous papers for more details where appropriate. In section II, we introduce the MAG and discuss its renormalizability when the operator $\left(\frac{1}{2} A_{\mu}^{a} A^{\mu a}+\alpha \bar{c}^{a} c^{a}\right)$ is introduced in the theory. We briefly review how the effective potential is constructed by means of the LCO technique. In section III, we discuss the independence of the vacuum energy from the gauge parameter of the MAG. We face the problem of the mixing of different orders in perturbation theory, and we provide a solution of it. In section IV, we construct a generalized renormalizable gauge that interpolates between the MAG and the Landau gauge. Moreover, we will also show that there exists a generalized renormalizable mass dimension two operator that interpolates between the mass dimension two operators of the MAG and of the Landau gauge. This can be used to prove that the vacuum energy obtained in the MAG is the same as that of the Landau gauge. In section $\mathrm{V}$, we present explicit results, obtained in the case of $S U(2)$ and to the one loop approximation. We end with conclusions in section VI.

\section{II. $S U(N)$ YANG-MILLS THEORIES IN THE MAG.}

Let $A_{\mu}$ be the Lie algebra valued connection for the gauge group $S U(N)$, whose generators $T^{A}$, satisfying $\left[T^{A}, T^{B}\right]=f^{A B C} T^{C}$, are chosen to be antihermitean and to obey the orthonormality condition $\operatorname{Tr}\left(T^{A} T^{B}\right)=$ $-T_{F} \delta^{A B}$, with $A, B, C=1, \ldots,\left(N^{2}-1\right)$. In the case of $S U(N)$, one has $T_{F}=\frac{1}{2}$. We decompose the gauge field into its off-diagonal and diagonal parts, namely

$$
A_{\mu}=A_{\mu}^{A} T^{A}=A_{\mu}^{a} T^{a}+A_{\mu}^{i} T^{i}
$$


where the indices $i, j, \ldots$ label the $N-1$ generators of the Cartan subalgebra. The remaining $N(N-1)$ off-diagonal generators will be labelled by the indices $a, b, \ldots$. For further use, we recall the Jacobi identity

$$
f^{A B C} f^{C D E}+f^{A D C} f^{C E B}+f^{A E C} f^{C B D}=0
$$

from which it can be deduced that

$$
\begin{aligned}
f^{a b i} f^{b j c}+f^{a b j} f^{b c i} & =0 \\
f^{a b c} f^{b d i}+f^{a b d} f^{b i c}+f^{a b i} f^{b c d} & =0 .
\end{aligned}
$$

The field strength decomposes as

$$
F_{\mu \nu}=F_{\mu \nu}^{A} T^{A}=F_{\mu \nu}^{a} T^{a}+F_{\mu \nu}^{i} T^{i}
$$

with the off-diagonal and diagonal parts given respectively by

$$
\begin{aligned}
& F_{\mu \nu}^{a}=D_{\mu}^{a b} A_{\nu}^{b}-D_{\nu}^{a b} A_{\mu}^{b}+g f^{a b c} A_{\mu}^{b} A_{\nu}^{c} \\
& F_{\mu \nu}^{i}=\partial_{\mu} A_{\nu}^{i}-\partial_{\nu} A_{\mu}^{i}+g f^{a b i} A_{\mu}^{a} A_{\nu}^{b}
\end{aligned}
$$

where the covariant derivative $D_{\mu}^{a b}$ is defined with respect to the diagonal components $A_{\mu}^{i}$

$$
D_{\mu}^{a b} \equiv \partial_{\mu} \delta^{a b}-g f^{a b i} A_{\mu}^{i}
$$

For the Yang-Mills action one obtains

$$
S_{\mathrm{YM}}=-\frac{1}{4} \int d^{4} x\left(F_{\mu \nu}^{a} F^{\mu \nu a}+F_{\mu \nu}^{i} F^{\mu \nu i}\right)
$$

The so called MAG gauge condition amounts to fixing the value of the covariant derivative, $D_{\mu}^{a b} A^{\mu b}$, of the off-diagonal components by requiring that the functional

$$
\mathcal{R}[A]=(V T)^{-1} \int d^{4} x\left(A_{\mu}^{a} A^{\mu a}\right)
$$

attains a minimum with respect to the local gauge transformations. This corresponds to imposing

$$
D_{\mu}^{a b} A^{\mu b}=0
$$

However, this condition being non-linear implies a quartic ghost self-interaction term is required for renormalizability purposes. The corresponding gauge fixing term turns out to be [8, [9]

$$
S_{\mathrm{MAG}}=s \int d^{4} x\left(\bar{c}^{a}\left(D_{\mu}^{a b} A^{b \mu}+\frac{\alpha}{2} b^{a}\right)-\frac{\alpha}{2} g f^{a b i} \bar{c}^{a} \bar{c}^{b} c^{i}-\frac{\alpha}{4} g f^{a b c} c^{a} \bar{c}^{b} \bar{c}^{c}\right),
$$

where $\alpha$ is the MAG gauge parameter and $s$ denotes the nilpotent BRST operator, acting as

$$
\begin{array}{rlrl}
s A_{\mu}^{a} & =-\left(D_{\mu}^{a b} c^{b}+g f^{a b c} A_{\mu}^{b} c^{c}+g f^{a b i} A_{\mu}^{b} c^{i}\right), & s A_{\mu}^{i}=-\left(\partial_{\mu} c^{i}+g f^{i a b} A_{\mu}^{a} c^{b}\right), \\
s c^{a}=g f^{a b i} c^{b} c^{i}+\frac{g}{2} f^{a b c} c^{b} c^{c}, & s c^{i}=\frac{g}{2} f^{i a b} c^{a} c^{b}, \\
s \bar{c}^{a}=b^{a}, & s \bar{c}^{i}=b^{i}, \\
s b^{a}=0, & s b^{i}=0 .
\end{array}
$$

Here $c^{a}, c^{i}$ are the off-diagonal and the diagonal components of the Faddeev-Popov ghost field, while $\bar{c}^{a}, b^{a}$ are the offdiagonal antighost and Lagrange multiplier. We also observe that the BRST transformations (11) have been obtained by their standard form upon projection on the off-diagonal and diagonal components of the fields. We remark that the MAG (10) can be written in the form

$$
S_{\mathrm{MAG}}=s \bar{s} \int d^{4} x\left(\frac{1}{2} A_{\mu}^{a} A^{\mu a}-\frac{\alpha}{2} c^{a} \bar{c}^{a}\right)
$$


with $\bar{s}$ being the nilpotent anti-BRST transformation, acting as

$$
\begin{aligned}
\bar{s} A_{\mu}^{a} & =-\left(D_{\mu}^{a b} \bar{c}^{b}+g f^{a b c} A_{\mu}^{b} \bar{c}^{c}+g f^{a b i} A_{\mu}^{b} \bar{c}^{i}\right), & & \bar{s} A_{\mu}^{i}=-\left(\partial_{\mu} \bar{c}^{i}+g f^{i a b} A_{\mu}^{a} \bar{c}^{b}\right), \\
\overline{s c}^{a} & =g f^{a b i} \bar{c}^{b} \bar{c}^{i}+\frac{g}{2} f^{a b c} \bar{c}^{b} \bar{c}^{c}, & & \overline{s c}^{i}=\frac{g}{2} f^{i a b} \bar{c}^{a} \bar{c}^{b}, \\
\bar{s} c^{a} & =-b^{a}+g f^{a b c} c^{b} \bar{c}^{c}+g f^{a b i} c^{b} \bar{c}^{i}+g f^{a b i} \bar{c}^{b} c^{i}, & & \bar{s} c^{i}=-b^{i}+g f^{i b c} c^{b} \bar{c}^{c}, \\
\bar{s} b^{a} & =-g f^{a b c} b^{b} \bar{c}^{c}-g f^{a b i} b^{b} \bar{c}^{i}+g f^{a b i} \bar{c}^{b} b^{i} & & \bar{s} b^{i}=-g f^{i b c} b^{b} \bar{c}^{c} .
\end{aligned}
$$

It can be checked that $s$ and $\bar{s}$ anticommute.

Expression (10) is easily worked out and yields

$$
\begin{aligned}
S_{\mathrm{MAG}} & =\int d^{4} x\left(b^{a}\left(D_{\mu}^{a b} A^{\mu b}+\frac{\alpha}{2} b^{a}\right)+\bar{c}^{a} D_{\mu}^{a b} D^{\mu b c} c^{c}+g \bar{c}^{a} f^{a b i}\left(D_{\mu}^{b c} A^{\mu c}\right) c^{i}+g \bar{c}^{a} D_{\mu}^{a b}\left(f^{b c d} A^{\mu c} c^{d}\right)\right. \\
& -\alpha g f^{a b i} b^{a} \bar{c}^{b} c^{i}-g^{2} f^{a b i} f^{c d i} \bar{c}^{a} c^{d} A_{\mu}^{b} A^{\mu c}-\frac{\alpha}{2} g f^{a b c} b^{a} \bar{c}^{b} c^{c}-\frac{\alpha}{4} g^{2} f^{a b i} f^{c d i} \bar{c}^{a} \bar{c}^{b} c^{c} c^{d} \\
& \left.-\frac{\alpha}{4} g^{2} f^{a b c} f^{a d i} \bar{c}^{b} \bar{c}^{c} c^{d} c^{i}-\frac{\alpha}{8} g^{2} f^{a b c} f^{a d e} \bar{c}^{b} \bar{c}^{c} c^{d} c^{e}\right) .
\end{aligned}
$$

We note that $\alpha=0$ does in fact correspond to the "real" MAG condition, given by eq. (9). However, one cannot set $\alpha=0$ from the beginning since this would lead to a nonrenormalizable gauge. Some of the terms proportional to $\alpha$ would reappear due to radiative corrections, even if $\alpha=0$. See, for example, [37]. For our purposes, this means that we have to keep $\alpha$ general throughout and leave to the end the analysis of the limit $\alpha \rightarrow 0$, to recover condition (9).

The MAG condition allows for a residual local $U(1)^{N-1}$ invariance with respect to the diagonal subgroup. In order to have a complete quantization of the theory, one has to fix this Abelian gauge freedom by means of a suitable further gauge condition on the diagonal components $A_{\mu}^{i}$ of the gauge field. A common choice for the Abelian gauge fixing, also adopted in the lattice papers [10, 11], is the Landau gauge, given by

$$
S_{\text {diag }}=s \int d^{4} x \bar{c}^{i} \partial_{\mu} A^{\mu i}=\int d^{4} x\left(b^{i} \partial_{\mu} A^{\mu i}+\bar{c}^{i} \partial^{\mu}\left(\partial_{\mu} c^{i}+g f^{i a b} A_{\mu}^{a} c^{b}\right)\right),
$$

where $\bar{c}^{i}, b^{i}$ are the diagonal antighost and Lagrange multiplier.

\section{A. Ward identities for the MAG.}

In order to write down a suitable set of Ward identities, we first introduce external fields $\Omega^{\mu i}, \Omega^{\mu a}, L^{i}, L^{a}$ coupled to the BRST nonlinear variations of the fields, namely

$$
\begin{aligned}
S_{\mathrm{ext}} & =\int d^{4} x\left(-\Omega^{\mu a}\left(D_{\mu}^{a b} c^{b}+g f^{a b c} A_{\mu}^{b} c^{c}+g f^{a b i} A_{\mu}^{b} c^{i}\right)-\Omega^{\mu i}\left(\partial_{\mu} c^{i}+g f^{i a b} A_{\mu}^{a} c^{b}\right)\right. \\
& \left.+L^{a}\left(g f^{a b i} c^{b} c^{i}+\frac{g}{2} f^{a b c} c^{b} c^{c}\right)+L^{i} \frac{g}{2} f^{i a b} c^{a} c^{b}\right),
\end{aligned}
$$

with

$$
\begin{aligned}
s \Omega^{\mu a} & =s \Omega^{\mu i}=0, \\
s L^{a} & =s L^{i}=0 .
\end{aligned}
$$

Moreover, in order to discuss the renormalizability of the gluon-ghost operator

$$
\mathcal{O}_{\mathrm{MAG}}=\frac{1}{2} A_{\mu}^{a} A^{\mu a}+\alpha \bar{c}^{a} c^{a},
$$

we introduce it in the starting action by means of a BRST doublet of external sources $(J, \lambda)$

$$
s \lambda=J, \quad s J=0,
$$

so that

$$
\begin{aligned}
S_{\mathrm{LCO}} & =s \int d^{4} x\left(\lambda\left(\frac{1}{2} A_{\mu}^{a} A^{\mu a}+\alpha \bar{c}^{a} c^{a}\right)+\zeta \frac{\lambda J}{2}\right) \\
& =\int d^{4} x\left(J\left(\frac{1}{2} A_{\mu}^{a} A^{\mu a}+\alpha \bar{c}^{a} c^{a}\right)+\zeta \frac{J^{2}}{2}-\alpha \lambda b^{a} c^{a}\right. \\
& \left.+\lambda A^{\mu a}\left(D_{\mu}^{a b} c^{b}+g f^{a b i} A_{\mu}^{b} c^{i}\right)+\alpha \lambda \bar{c}^{a}\left(g f^{a b i} c^{b} c^{i}+\frac{g}{2} f^{a b c} c^{b} c^{c}\right)\right),
\end{aligned}
$$




\begin{tabular}{|c|c|c|c|c|c|c|c|c|}
\hline & $A_{\mu}^{a, \imath}$ & $c^{a, \imath}$ & $\bar{c}^{a, 2}$ & $b^{a, \imath}$ & $\lambda$ & $J$ & $\Omega_{\mu}^{a, 2}$ & $L^{a, \imath}$ \\
\hline dimension & 1 & 0 & 2 & 2 & 2 & 2 & 3 & 4 \\
ghost number & 0 & 1 & -1 & 0 & -1 & 0 & -1 & -2 \\
\hline
\end{tabular}

TABLE I: Dimension and ghost number.

where $\zeta$ is the LCO parameter accounting for the divergences present in the vacuum correlator $\left\langle\mathcal{O}_{\mathrm{MAG}}(x) \mathcal{O}_{\mathrm{MAG}}(y)\right\rangle$, which are proportional to $J^{2}$. Therefore, the complete action

$$
\Sigma=S_{\mathrm{YM}}+S_{\mathrm{MAG}}+S_{\mathrm{diag}}+S_{\mathrm{ext}}+S_{\mathrm{LCO}},
$$

is BRST invariant

$$
s \Sigma=0 .
$$

As noticed in [16, 38], the gluon-ghost mass operator defined in eq.(18) is BRST invariant on-shell.

In Table I, the dimension and ghost number of all the fields and sources are listed. We are now ready to write down the Ward identities needed to discuss the renormalizability of the model. It turns out that the complete action $\Sigma$ is constrained by

- the Slavnov-Taylor identity

$$
\mathcal{S}(\Sigma)=0
$$

with

$$
\mathcal{S}(\Sigma)=\int d^{4} x\left(\frac{\delta \Sigma}{\delta \Omega^{\mu a}} \frac{\delta \Sigma}{\delta A_{\mu}^{a}}+\frac{\delta \Sigma}{\delta \Omega^{\mu i}} \frac{\delta \Sigma}{\delta A_{\mu}^{i}}+\frac{\delta \Sigma}{\delta L^{a}} \frac{\delta \Sigma}{\delta c^{a}}+\frac{\delta \Sigma}{\delta L^{i}} \frac{\delta \Sigma}{\delta c^{i}}+b^{a} \frac{\delta \Sigma}{\delta \bar{c}^{a}}+b^{i} \frac{\delta \Sigma}{\delta \bar{c}^{i}}+J \frac{\delta \Sigma}{\delta \lambda}\right)
$$

- the diagonal ghost equation [9]

$$
\mathcal{G}^{i} \Sigma=\Delta_{\mathrm{cl}}^{i}
$$

where

$$
\mathcal{G}^{i}=\frac{\delta}{\delta c^{i}}+g f^{a b i} \bar{c}^{a} \frac{\delta \Sigma}{\delta b^{b}}
$$

and

$$
\Delta_{\mathrm{cl}}^{i}=-\partial^{2} \bar{c}^{i}+g f^{a b i} \Omega^{\mu a} A_{\mu}^{b}-\partial_{\mu} \Omega^{\mu i}-g f^{a b i} L^{a} c^{b} .
$$

Notice that expression (27), being linear in the quantum fields, is a classical breaking.

- the diagonal gauge fixing and anti-ghost equations

$$
\begin{aligned}
\frac{\delta \Sigma}{\delta b^{i}} & =\partial_{\mu} A^{\mu i}, \\
\frac{\delta \Sigma}{\delta \bar{c}^{i}}+\partial^{\mu} \frac{\delta \Sigma}{\delta \Omega^{\mu i}} & =0 .
\end{aligned}
$$

- the integrated $\lambda$-equation

$$
\int d^{4} x\left(\frac{\delta \Sigma}{\delta \lambda}+c^{a} \frac{\delta \Sigma}{\delta b^{a}}\right)=0
$$

expressing in a functional form the on-shell BRST invariance of the gluon-ghost operator $\mathcal{O}_{\mathrm{MAG}}$.

- the diagonal $U(1)^{N-1}$ Ward identity

$$
\mathcal{W}^{i} \Sigma=-\partial^{2} b^{i}
$$

with

$$
\mathcal{W}^{i}=\partial_{\mu} \frac{\delta}{\delta A_{\mu}^{i}}+g f^{a b i}\left(A_{\mu}^{a} \frac{\delta}{\delta A_{\mu}^{b}}+c^{a} \frac{\delta}{\delta c^{b}}+b^{a} \frac{\delta}{\delta b^{b}}+\bar{c}^{a} \frac{\delta}{\delta \bar{c}^{b}}+\Omega^{\mu a} \frac{\delta}{\delta \Omega^{\mu b}}+L^{a} \frac{\delta \Sigma}{\delta L^{b}}\right) .
$$

This identity follows from the diagonal ghost equation (25) and the Slavnov-Taylor identity (23).

In order to find the foregoing Ward identities, use has been made of the Jacobi identities (3). 


\section{B. Algebraic characterization of the most general local counterterm.}

We mention that all the classical Ward identities of the previous section can be extended to all orders of perturbation theory without encountering anomalies. In principle, this can be proven by means of the algebraic setup of [30] and of the general results on the BRST cohomology of gauge theories [39]. It can be understood in a simple way by observing that pure Yang-Mills theory in the MAG can be regularized in a gauge invariant way by employing dimensional regularization.

In order to characterize the most general invariant counterterm which can be freely added to all orders of perturbation theory, we perturb the classical action $\Sigma$ by adding an arbitrary integrated local polynomial $\Sigma^{\text {count }}$ in the fields and external sources of dimension bounded by four and with zero ghost number, and we require that the perturbed action $\left(\Sigma+\eta \Sigma^{\text {count }}\right)$ satisfies the same Ward identities as $\Sigma$ to the first order in the perturbation parameter $\eta$, i.e.,

$$
\begin{aligned}
\mathcal{S}\left(\Sigma+\eta \Sigma^{\text {count }}\right) & =0+O\left(\eta^{2}\right), \\
\mathcal{G}^{i}\left(\Sigma+\eta \Sigma^{\text {count }}\right) & =\Delta_{\mathrm{cl}}^{i}+O\left(\eta^{2}\right), \\
\frac{\delta\left(\Sigma+\eta \Sigma^{\text {count }}\right)}{\delta b^{i}} & =\partial_{\mu} A^{\mu i}+O\left(\eta^{2}\right), \\
\left(\frac{\delta}{\delta \bar{c}^{i}}+\partial^{\mu} \frac{\delta}{\delta \Omega^{\mu i}}\right)\left(\Sigma+\eta \Sigma^{\text {count }}\right) & =0+O\left(\eta^{2}\right), \\
\int d^{4} x\left(\frac{\delta}{\delta \lambda}+c^{a} \frac{\delta}{\delta b^{a}}\right)\left(\Sigma+\eta \Sigma^{\text {count }}\right) & =0+O\left(\eta^{2}\right), \\
\mathcal{W}^{i}\left(\Sigma+\eta \Sigma^{\text {count }}\right) & =-\partial^{2} b^{i}+O\left(\eta^{2}\right) .
\end{aligned}
$$

This amounts to imposing the following conditions on $\Sigma^{\text {count }}$

$$
\mathcal{B}_{\Sigma} \Sigma^{\text {count }}=0
$$

where $\mathcal{B}_{\Sigma}$ denotes the nilpotent linearized operator

$$
\begin{gathered}
\mathcal{B}_{\Sigma} \mathcal{B}_{\Sigma}=0, \\
\mathcal{B}_{\Sigma}=\int d^{4} x\left(\frac{\delta \Sigma}{\delta \Omega^{\mu a}} \frac{\delta}{\delta A_{\mu}^{a}}+\frac{\delta \Sigma}{\delta A_{\mu}^{a}} \frac{\delta}{\delta \Omega^{\mu a}}+\frac{\delta \Sigma}{\delta \Omega^{\mu i}} \frac{\delta}{\delta A_{\mu}^{i}}+\frac{\delta \Sigma}{\delta A_{\mu}^{i}} \frac{\delta}{\delta \Omega^{\mu i}}+\frac{\delta \Sigma}{\delta L^{a}} \frac{\delta}{\delta c^{a}}\right. \\
\left.+\frac{\delta \Sigma}{\delta c^{a}} \frac{\delta}{\delta L^{a}}+\frac{\delta \Sigma}{\delta L^{i}} \frac{\delta}{\delta c^{i}}+\frac{\delta \Sigma}{\delta c^{i}} \frac{\delta}{\delta L^{i}}+b^{a} \frac{\delta}{\delta \bar{c}^{a}}+b^{i} \frac{\delta}{\delta \bar{c}^{i}}+J \frac{\delta}{\delta \lambda}\right),
\end{gathered}
$$

and

$$
\begin{aligned}
\mathcal{G}^{i} \Sigma^{\text {count }} & =0, \\
\frac{\delta \Sigma^{\text {count }}}{\delta b^{i}} & =0, \\
\left(\frac{\delta}{\delta \bar{c}^{i}}+\partial^{\mu} \frac{\delta}{\delta \Omega^{\mu i}}\right) \Sigma^{\text {count }} & =0, \\
\int d^{4} x\left(\frac{\delta}{\delta \lambda}+c^{a} \frac{\delta}{\delta b^{a}}\right) \Sigma+\varepsilon \Sigma^{\text {count }} & =0, \\
\mathcal{W}^{i} \Sigma^{\text {count }} & =0 .
\end{aligned}
$$

¿From the conditions (33) and (36), it turns out that the most general invariant counterterm can be written as

$$
\Sigma^{\text {count }}=\frac{-a_{0}}{4} \int d^{4} x\left(F_{\mu \nu}^{a} F^{\mu \nu a}+F_{\mu \nu}^{i} F^{\mu \nu i}\right)+\mathcal{B}_{\Sigma} \Delta^{-1},
$$

where $\Delta^{-1}$ is an integrated local polynomial with ghost number -1 , given by

$$
\begin{aligned}
\Delta^{-1} & =\int d^{4} x\left(a_{1} L^{a} c^{a}+a_{3} \Omega^{\mu a} A_{\mu}^{a}+a_{5} \bar{c}^{a}\left(b^{a}-g f^{a b i} \bar{c}^{b} c^{i}\right)+a_{6} \bar{c}^{a} D_{\mu}^{a b} A^{\mu b}-\frac{a_{5}}{2} g f^{a b c} \bar{c}^{a} \bar{c}^{b} c^{c}\right. \\
& \left.+a_{1} \lambda\left(\frac{1}{2} A_{\mu}^{a} A^{\mu a}+\alpha \bar{c}^{a} c^{a}\right)+\frac{a_{6}}{2} \lambda A_{\mu}^{a} A^{\mu a}+2 \alpha a_{5} \lambda \bar{c}^{a} c^{a}+\frac{a_{13} \zeta}{2} \lambda J\right) .
\end{aligned}
$$


We see thus that $\Sigma^{\text {count }}$ contains six free independent parameters, namely $a_{0}, a_{1}, a_{3}, a_{5}, a_{6}$ and $a_{13}$. These parameters can be reabsorbed by means of a multiplicative renormalization of the gauge coupling constant $g$, of the gauge and LCO parameters $\alpha, \zeta$, and of the fields $\phi=\left(A^{\mu a}, A^{\mu i}, c^{a}, \bar{c}^{a}, c^{i}, \bar{c}^{i}, b^{a}, b^{i}\right)$ and sources $\Phi=\left(\Omega^{\mu a}, \Omega^{\mu i}, L^{a}, L^{i}, \lambda, J\right)$, according to

$$
\Sigma(g, \alpha, \zeta, \phi, \Phi)+\eta \Sigma^{\mathrm{count}}=\Sigma\left(g_{0}, \alpha_{0}, \zeta_{0}, \phi_{0}, \Phi_{0}\right)+O\left(\eta^{2}\right),
$$

with

$$
\begin{gathered}
g_{0}=Z_{g} g, \quad \alpha_{0}=Z_{\alpha} \alpha, \quad \zeta_{0}=Z_{\zeta} \zeta, \\
A_{0}^{\mu a}=\widetilde{Z}_{A}^{1 / 2} A^{\mu a}, \quad A_{0}^{\mu i}=Z_{g}^{-1} A^{\mu i}, \\
c_{0}^{a}=\widetilde{Z}_{c}^{1 / 2} c^{a}, \quad \bar{c}_{0}^{a}=\widetilde{Z}_{c}^{1 / 2} \bar{c}^{a}, \\
c_{0}^{i}=Z_{c}^{1 / 2} c^{i}, \quad \bar{c}_{0}^{i}=Z_{c}^{-1 / 2} \bar{c}^{i}, \\
b_{0}^{a}=Z_{g} Z_{c}^{1 / 2} \widetilde{Z}_{c}^{1 / 2} b^{a}, \quad b_{0}^{i}=Z_{g} b^{i}, \\
\Omega_{0}^{\mu a}=\widetilde{Z}_{A}^{-1 / 2} Z_{g}^{-1} Z_{c}^{-1 / 2} \Omega^{\mu a}, \quad \Omega_{0}^{\mu i}=Z_{c}^{-1 / 2} \Omega^{\mu i}, \\
L_{0}^{a}=Z_{g}^{-1} \widetilde{Z}_{c}^{-1 / 2} Z_{c}^{-1 / 2} L^{a}, \quad L_{0}^{i}=Z_{g}^{-1} Z_{c}^{-1} L^{i},
\end{gathered}
$$

and

$$
\begin{aligned}
& J_{0}=Z_{L^{a}}^{-2} \widetilde{Z}_{c}^{-1} J=Z_{g}^{2} Z_{c} J, \\
& \lambda_{0}=Z_{L^{a}}^{-1} \widetilde{Z}_{c}^{-1 / 2} \lambda=Z_{g} Z_{c}^{1 / 2} \lambda,
\end{aligned}
$$

with

$$
\begin{aligned}
Z_{g} & =1-\eta \frac{a_{0}}{2}, \\
Z_{\alpha} & =1+\eta\left(\frac{2 a_{5}}{\alpha}+a_{0}-2 a_{6}\right), \\
Z_{\zeta} & =1+\eta\left(a_{13}+2 a_{0}-2 a_{1}-2 a_{6}\right), \\
\widetilde{Z}_{A}^{1 / 2} & =1+\eta\left(\frac{a_{0}}{2}+a_{3}\right), \\
\widetilde{Z}_{c}^{1 / 2} & =1+\frac{\eta}{2}\left(a_{6}-a_{1}\right), \\
Z_{c}^{1 / 2} & =1+\frac{\eta}{2}\left(a_{6}+a_{1}\right) .
\end{aligned}
$$

In particular, from eq. (46), one sees that the renormalization of the source $J$, and thus of the composite operator $\mathcal{O}_{\mathrm{MAG}}$, can be expressed in terms of the renormalization of gauge coupling constant and of the diagonal ghost. This property follows from the diagonal ghost equation (25) and from the the integrated $\lambda$-equation (29). In particular, for the anomalous dimension of the gluon-ghost operator $\mathcal{O}_{\mathrm{MAG}}$, we obtain [19]

$$
\gamma_{\mathcal{O}_{\mathrm{MAG}}}\left(g^{2}\right)=\mu \frac{\partial}{\partial \mu} \log \left(Z_{g}^{2} Z_{c}\right)=-2\left(\frac{\beta\left(g^{2}\right)}{2 g^{2}}-\gamma_{c^{i}}\left(g^{2}\right)\right),
$$

with

$$
\begin{aligned}
\beta\left(g^{2}\right) & =\mu \frac{\partial g^{2}}{\partial \mu}=-g^{2} \mu \frac{\partial}{\partial \mu} \ln Z_{g}^{2}, \\
\gamma_{c^{i}}\left(g^{2}\right) & =\mu \frac{\partial}{\partial \mu} \ln Z_{c}^{1 / 2} .
\end{aligned}
$$




\section{The effective potential.}

We present here the main steps in the construction of the effective potential for a local composite operator. A more detailed account of the LCO formalism can be found in [40, 41].

To obtain the effective potential for the condensate $\left\langle\mathcal{O}_{\mathrm{MAG}}\right\rangle$, we set the sources $\Omega_{\mu}^{i}, \Omega_{\mu}^{a}, L^{a}, L^{i}$ and $\lambda$ to zero and consider the renormalized generating functional

$$
\begin{aligned}
\exp (-i \mathcal{W}(J)) & =\int[D \varphi] \exp i S(J) \\
S(J) & =S_{\mathrm{YM}}+S_{\mathrm{MAG}}+S_{\mathrm{diag}}+S_{\mathrm{count}}+\int d^{4} x\left(Z_{J} J\left(\frac{1}{2} \widetilde{Z}_{A} A_{\mu}^{a} A^{\mu a}+Z_{\alpha} \widetilde{Z}_{c} \alpha \bar{c}^{a} c^{a}\right)+(\zeta+\delta \zeta) \frac{J^{2}}{2}\right)
\end{aligned}
$$

where $\varphi$ denotes the relevant fields and $S_{\text {count }}$ is the usual counterterm contribution, i.e. the part without the composite operator. The quantity $\delta \zeta$ is the counterterm accounting for the divergences proportional to $J^{2}$. Using dimensional regularization throughout with the convention that $d=4-\varepsilon$, one has the following identification

$$
\zeta_{0} J_{0}^{2}=\mu^{-\varepsilon}(\zeta+\delta \zeta) J^{2}
$$

The functional $\mathcal{W}(J)$ obeys the renormalization group equation (RGE)

$$
\left(\mu \frac{\partial}{\partial \mu}+\beta\left(g^{2}\right) \frac{\partial}{\partial g^{2}}+\alpha \gamma_{\alpha}\left(g^{2}\right) \frac{\partial}{\partial \alpha}-\gamma_{\mathcal{O}_{\mathrm{MAG}}}\left(g^{2}\right) \int d^{4} x J \frac{\delta}{\delta J}+\eta\left(g^{2}, \zeta\right) \frac{\partial}{\partial \zeta}\right) \mathcal{W}(J)=0
$$

where

$$
\begin{aligned}
\gamma_{\alpha}\left(g^{2}\right) & =\mu \frac{\partial}{\partial \mu} \ln \alpha=\mu \frac{\partial}{\partial \mu} \ln Z_{\alpha}^{-1}, \\
\eta\left(g^{2}, \zeta\right) & =\mu \frac{\partial}{\partial \mu} \zeta .
\end{aligned}
$$

¿From eq.(51), one finds

$$
\eta\left(g^{2}, \zeta\right)=2 \gamma_{\mathcal{O}_{\mathrm{MAG}}}\left(g^{2}\right) \zeta+\delta\left(g^{2}, \alpha\right)
$$

with

$$
\delta\left(g^{2}, \alpha\right)=\left(\varepsilon+2 \gamma_{\mathcal{O}_{\mathrm{MAG}}}\left(g^{2}\right)-\beta\left(g^{2}\right) \frac{\partial}{\partial g^{2}}-\alpha \gamma_{\alpha}\left(g^{2}\right) \frac{\partial}{\partial \alpha}\right) \delta \zeta
$$

Up to now, the LCO parameter $\zeta$ is still an arbitrary coupling. As explained in [40, 41], simply setting $\zeta=0$ would give rise to an inhomogeneous $\mathrm{RGE}$ for $\mathcal{W}(J)$

$$
\left(\mu \frac{\partial}{\partial \mu}+\beta\left(g^{2}\right) \frac{\partial}{\partial g^{2}}+\alpha \gamma_{\alpha}\left(g^{2}\right) \frac{\partial}{\partial \alpha}-\gamma_{\mathcal{O}_{\mathrm{MAG}}}\left(g^{2}\right) \int d^{4} x J \frac{\delta}{\delta J}\right) \mathcal{W}(J)=\delta\left(g^{2}, \alpha\right) \int d^{4} x \frac{J^{2}}{2},
$$

and a non-linear RGE for the associated effective action $\Gamma$ for the composite operator $\mathcal{O}_{\mathrm{MAG}}$. Furthermore, multiplicative renormalizability is lost and by varying the value of $\delta \zeta$, minima of the effective action can change into maxima or can get lost. However, $\zeta$ can be made such a function of $g^{2}$ and $\alpha$ so that, if $g^{2}$ runs according to $\beta\left(g^{2}\right)$ and $\alpha$ according to $\gamma_{\alpha}\left(g^{2}\right), \zeta\left(g^{2}, \alpha\right)$ will run according to its RGE (54). This is accomplished by setting $\zeta$ equal to the solution of the differential equation

$$
\left(\beta\left(g^{2}\right) \frac{\partial}{\partial g^{2}}+\alpha \gamma_{\alpha}\left(g^{2}, \alpha\right) \frac{\partial}{\partial \alpha}\right) \zeta\left(g^{2}, \alpha\right)=2 \gamma_{\mathcal{O}_{\mathrm{MAG}}}\left(g^{2}\right) \zeta\left(g^{2}, \alpha\right)+\delta\left(g^{2}, \alpha\right)
$$

Doing so, $\mathcal{W}(J)$ obeys the homogeneous renormalization group equation

$$
\left(\mu \frac{\partial}{\partial \mu}+\beta\left(g^{2}\right) \frac{\partial}{\partial g^{2}}+\alpha \gamma_{\alpha}\left(g^{2}\right) \frac{\partial}{\partial \alpha}-\gamma_{\mathcal{O}_{\mathrm{MAG}}}\left(g^{2}\right) \int d^{4} x J \frac{\delta}{\delta J}\right) \mathcal{W}(J)=0 .
$$

To lighten the notation, we will drop the renormalization factors from now on. One will notice that there are terms quadratic in the source $J$ present in $\mathcal{W}(J)$, obscuring the usual energy interpretation. This can be cured by removing 
the terms proportional to $J^{2}$ in the action to get a generating functional that is linear in the source, a goal easily achieved by inserting the following unity,

$$
1=\frac{1}{N} \int[D \sigma] \exp \left[i \int d^{4} x\left(-\frac{1}{2 \zeta}\left(\frac{\sigma}{g}-\mathcal{O}_{\mathrm{MAG}}-\zeta J\right)^{2}\right)\right],
$$

with $N$ the appropriate normalization factor, in eq.(50) to arrive at the Lagrangian

$$
\mathcal{L}\left(A_{\mu}, \sigma\right)=-\frac{1}{4} F_{\mu \nu}^{a} F^{\mu \nu a}-\frac{1}{4} F_{\mu \nu}^{i} F^{\mu \nu i}+\mathcal{L}_{\mathrm{MAG}}+\mathcal{L}_{\mathrm{diag}}-\frac{\sigma^{2}}{2 g^{2} \zeta}+\frac{1}{g^{2} \zeta} g \sigma \mathcal{O}_{\mathrm{MAG}}-\frac{1}{2 \zeta}\left(\mathcal{O}_{\mathrm{MAG}}\right)^{2},
$$

while

$$
\begin{aligned}
\exp (-i \mathcal{W}(J)) & =\int[D \varphi] \exp i S_{\sigma}(J) \\
S_{\sigma}(J) & =\int d^{4} x\left(\mathcal{L}\left(A_{\mu}, \sigma\right)+J \frac{\sigma}{g}\right) .
\end{aligned}
$$

¿From eqs.(50) and (61), one has the following simple relation

$$
\left.\frac{\delta \mathcal{W}(J)}{\delta J}\right|_{J=0}=-\left\langle\mathcal{O}_{\mathrm{MAG}}\right\rangle=-\left\langle\frac{\sigma}{g}\right\rangle
$$

meaning that the condensate $\left\langle\mathcal{O}_{\mathrm{MAG}}\right\rangle$ is directly related to the expectation value of the field $\sigma$, evaluated with the action $S_{\sigma}=\int d^{4} x \mathcal{L}\left(A_{\mu}, \sigma\right)$. As it is obvious from eq. (60), $\langle\sigma\rangle \neq 0$ is sufficient to have a tree level dynamical mass for the off-diagonal fields. At lowest order (i.e. tree level), one finds

$$
\begin{aligned}
& m_{\text {gluon }}^{\text {off-diag. }}=\sqrt{\frac{g \sigma}{\zeta_{0}}}, \\
& m_{\text {ghost }}^{\text {off }- \text { diag. }}=\sqrt{\alpha \frac{g \sigma}{\zeta_{0}}} .
\end{aligned}
$$

Meanwhile, the diagonal degrees of freedom remain massless. This could have been established already from the local $U(1)^{N-1}$ Ward identity (301).

\section{GAUGE PARAMETER INDEPENDENCE OF THE VACUUM ENERGY.}

We begin this section with a few remarks on the determination of $\zeta\left(g^{2}, \alpha\right)$. From explicit calculations in perturbation theory, it will become clear [55] that the RGE functions showing up in the differential equation (57) look like

$$
\begin{aligned}
\beta\left(g^{2}\right) & =-\varepsilon g^{2}-2\left(\beta_{0} g^{2}+\beta_{1} g^{2}+\cdots\right), \\
\gamma_{\mathcal{O}_{\mathrm{MAG}}}\left(g^{2}\right) & =\gamma_{0}(\alpha) g^{2}+\gamma_{1}(\alpha) g^{4}+\cdots, \\
\gamma_{\alpha}\left(g^{2}\right) & =a_{0}(\alpha) g^{2}+a_{1}(\alpha) g^{4}+\cdots, \\
\delta\left(g^{2}, \alpha\right) & =\delta_{0}(\alpha)+\delta_{1}(\alpha) g^{2}+\cdots .
\end{aligned}
$$

As such, eq.(57) can be solved by expanding $\zeta\left(g^{2}, \alpha\right)$ in a Laurent series in $g^{2}$,

$$
\zeta\left(g^{2}, \alpha\right)=\frac{\zeta_{0}(\alpha)}{g^{2}}+\zeta_{1}(\alpha)+\zeta_{2}(\alpha) g^{2}+\cdots
$$

More precisely, for the first coefficients $\zeta_{0}, \zeta_{1}$ of the expression (66), one obtains

$$
\begin{aligned}
2 \beta_{0} \zeta_{0}+\alpha a_{0} \frac{\partial \zeta_{0}}{\partial \alpha} & =2 \gamma_{0} \zeta_{0}+\delta_{0} \\
2 \beta_{1} \zeta_{0}+\alpha a_{0} \frac{\partial \zeta_{1}}{\partial \alpha}+\alpha a_{1} \frac{\partial \zeta_{0}}{\partial \alpha} & =2 \gamma_{0} \zeta_{1}+2 \gamma_{1} \zeta_{0}+\delta_{1}
\end{aligned}
$$


Notice that, in order to construct the $n$-loop effective potential, knowledge of the $(n+1)$-loop RGE functions is needed.

The effective potential calculated with the Lagrangian (60) will explicitly depend on the gauge parameter $\alpha$. The question arises concerning the vacuum energy $E_{\mathrm{vac}}$, (i.e. the effective potential evaluated at its minimum); will it be independent of the choice of $\alpha$ ? Also, as it can be seen from the equations [67), each $\zeta_{i}(\alpha)$ is determined through a first order differential equation in $\alpha$. Firstly, one has to solve for $\zeta_{0}(\alpha)$. This will introduce one arbitrary integration constant $C_{0}$. Using the obtained value for $\zeta_{0}(\alpha)$, one can consequently solve the first order differential equation for $\zeta_{1}(\alpha)$. This will introduce a second integration constant $C_{1}$, etc. In principle, it is possible that these arbitrary constants influence the vacuum energy, which would represent an unpleasant feature. Notice that the differential equations in $\alpha$ for the $\zeta_{i}$ are due to the running of $\alpha$ in eq.(57), encoded in the renormalization group function $\gamma_{\alpha}\left(g^{2}\right)$. Assume that we would have already shown that $E_{\mathrm{vac}}$ does not depend on the choice of $\alpha$. If we then set $\alpha=\alpha^{*}$, with $\alpha^{*}$ a fixed point of the RGE for $\alpha$ at the considered order of perturbation theory, then equation (57) determining $\zeta$ simplifies to

$$
\beta\left(g^{2}\right) \frac{\partial}{\partial g^{2}} \zeta\left(g^{2}, \alpha^{*}\right)=2 \gamma_{\mathcal{O}_{\mathrm{MAG}}}\left(g^{2}\right) \zeta\left(g^{2}, \alpha^{*}\right)+\delta\left(g^{2}, \alpha^{*}\right)
$$

since

$$
\left.\gamma_{\alpha}\left(g^{2}\right) \alpha\right|_{\alpha=\alpha^{*}}=0
$$

This will lead to simple algebraic equations for the $\zeta_{i}\left(\alpha^{*}\right)$. Hence, no integration constants will enter the final result for the vacuum energy for $\alpha=\alpha^{*}$, and since $E_{\mathrm{vac}}$ does not depend on $\alpha, E_{\mathrm{vac}}$ will never depend on the integration constants, even when calculated for a general $\alpha$. Hence, we can put them equal to zero from the beginning for simplicity.

Summarizing, two questions remain. Firstly, we should prove that the value of $\alpha$ will not influence the obtained value for $E_{\mathrm{vac}}$. Secondly, we should show that there exists a fixed point $\alpha^{*}$. We postpone the discussion concerning the second question to the next section, giving a positive answer to the first one. In order to do so, let us reconsider the generating functional (61). We have the following identification, ignoring the overall normalization factors

$$
\exp (-i \mathcal{W}(J))=\int[D \varphi] \exp i S_{\sigma}(J)=\frac{1}{N} \int[D \varphi D \sigma] \exp i\left[S(J)+\int d^{4} x\left(-\frac{1}{2 \zeta}\left(\frac{\sigma}{g}-\mathcal{O}_{\mathrm{MAG}}-\zeta J\right)^{2}\right)\right],
$$

where $S(J)$ and $S_{\sigma}(J)$ are given respectively by eq.(50), and eq.(62). Obviously,

$$
\frac{d}{d \alpha} \frac{1}{N} \int[D \sigma] \exp \left[i \int d^{4} x\left(-\frac{1}{2 \zeta}\left(\frac{\sigma}{g}-\mathcal{O}_{\mathrm{MAG}}-\zeta J\right)^{2}\right)\right]=\frac{d}{d \alpha} 1=0,
$$

so that

$$
\frac{d \mathcal{W}(J)}{d \alpha}=-\left.\left\langle s \int d^{4} x \bar{s}\left(\frac{1}{2} c^{a} \bar{c}^{a}\right)\right\rangle\right|_{J=0}+\text { terms } \propto J
$$

which follows directly from

$$
\frac{d S(J)}{d \alpha}=s \bar{s} \int d^{4} x\left(\frac{1}{2} c^{a} \bar{c}^{a}\right)+\text { terms } \propto J .
$$

We see that the first term in the right hand side of (73) is an exact BRST variation. As such, its vacuum expectation value vanishes. This is the usual argument to prove the gauge parameter independence in the BRST framework [30]. Note that no local operator $\hat{\mathcal{O}}$, with $s \hat{\mathcal{O}}=\mathcal{O}_{\mathrm{MAG}}$, exists. Furthermore, extending the action of the BRST transformation on the $\sigma$-field by

$$
s \sigma=g s \mathcal{O}_{\mathrm{MAG}}=-A^{\mu a} D_{\mu}^{a b} c^{b}+\alpha b^{a} c^{a}-\alpha g f^{a b i} \bar{c}^{a} \bar{c}^{b} c^{i}-\frac{\alpha}{2} g f^{a b c} \bar{c}^{a} c^{b} c^{c}
$$

one can easily check that

$$
s \int d^{4} x \mathcal{L}\left(A_{\mu}, \sigma\right)=0,
$$


so that we have a BRST invariant $\sigma$-action. Thus, when we consider the vacuum, corresponding to $J=0$, only the BRST exact term in eq.(72) survives. The effective action $\Gamma$ is related to $\mathcal{W}(J)$ through a Legendre transformation

$$
\Gamma\left(\frac{\sigma}{g}\right)=-\mathcal{W}(J)-\int d^{4} y J(y) \frac{\sigma(y)}{g} .
$$

The effective potential $V(\sigma)$ is then defined as

$$
-V(\sigma) \int d^{4} x=\Gamma\left(\frac{\sigma}{g}\right) .
$$

Let $\sigma_{\min }$ be the solution of

$$
\frac{d V(\sigma)}{d \sigma}=0
$$

From

$$
\frac{\delta}{\delta\left(\frac{\sigma}{g}\right)} \Gamma=-J
$$

it follows that

$$
\sigma=\sigma_{\min } \Rightarrow J=0
$$

and hence, we derive from eqs. (76) and (77) that

$$
\left.\frac{d}{d \alpha} V(\sigma)\right|_{\sigma=\sigma_{\min }} \int d^{4} x=\left.\frac{d}{d \alpha} \mathcal{W}(J)\right|_{J=0} .
$$

Thus, due to eq.(72),

$$
\left.\frac{d}{d \alpha} V(\sigma)\right|_{\sigma=\sigma_{\min }}=0 .
$$

We conclude that the vacuum energy $E_{\mathrm{vac}}$ should be independent from the gauge parameter $\alpha$.

A completely analogous derivation was obtained in the case of the linear gauge [33]. Nevertheless, in spite of the previous argument, explicit results in that case showed that $E_{\text {vac }}$ did depend on $\alpha$. In [33] it was argued that this apparent disagreement was due to a mixing of different orders of perturbation theory. Let us explain this with a simple example. Let us first notice that a key argument in the previous analysis is that the source $J=0$ vanishes at the end of the calculations. In practice, $J=0$ is achieved by solving the gap equation (78). Moreover, in a power series expansion in the coupling constant, the derivative of the effective potential with respect to $\sigma$ will look like

$$
\left(v_{0}+v_{1} g^{2}+O\left(g^{4}\right)\right) \sigma
$$

where we assume that we work up to order $g^{2}$. The corresponding gap equation reads

$$
v_{0}+v_{1} g^{2}+O\left(g^{4}\right)=0
$$

Due to eqs.(77) and (79), one also has

$$
J=g\left(v_{0}+v_{1} g^{2}+O\left(g^{4}\right)\right) \sigma
$$

Imposing the gap equation (84) leads to

$$
J=g\left(0+O\left(g^{4}\right)\right) \sigma
$$

However, as it can be immediately checked from expression (70), there are several terms proportional to $J$ in the right-hand side of eq.(72). For instance, one of them is given by $\frac{\partial \zeta}{\partial \alpha} J^{2}$. Since

$$
\frac{\partial \zeta}{\partial \alpha}=\frac{\partial \zeta_{0}}{\partial \alpha} \frac{1}{g^{2}}+\frac{\partial \zeta_{1}}{\partial \alpha}+O\left(g^{2}\right) .
$$


we find

$$
\frac{\partial \zeta}{\partial \alpha} J^{2}=\left(\frac{\partial \zeta_{0}}{\partial \alpha} v_{0}^{2}+\left(\frac{\partial \zeta_{0}}{\partial \alpha} 2 v_{0} v_{1}+\frac{\partial \zeta_{1}}{\partial \alpha} v_{0}^{2}\right) g^{2}+O\left(g^{4}\right)\right) \sigma^{2} .
$$

Squaring the gap equation (84),

$$
v_{0}^{2}+2 v_{1} v_{0} g^{2}+O\left(g^{4}\right)=0,
$$

leads to

$$
\frac{\partial \zeta}{\partial \alpha} J^{2}=\left(\frac{\partial \zeta_{1}}{\partial \alpha} v_{0}^{2} g^{2}+O\left(g^{4}\right)\right) \sigma^{2}
$$

We see that, if one consistently works to the first order, terms such as $\frac{\partial \zeta}{\partial \alpha} J^{2}$ do not equal zero, although $J=0$ to that order. Terms like those on the right-hand side of eq.90) are cancelled by terms which are formally of higher order, requiring thus a mixing of different orders of perturbation theory. Of course, this problem would not have occurred if we were be able to compute the effective potential up to infinite order, an evidently hopeless task. Nevertheless, in [33. we succeeded in finding a suitable modification of the LCO formalism in order to circumvent this problem and obtaining a well defined gauge independent vacuum energy $E_{\mathrm{vac}}$, without the need of working at infinite order. Instead of the action (50), let us consider the following action

$$
\widetilde{S}(\widetilde{J})=S_{\mathrm{YM}}+S_{\mathrm{MAG}}+S_{\mathrm{diag}}+\int d^{4} x\left[\widetilde{J} \mathcal{F}\left(g^{2}, \alpha\right) \mathcal{O}_{\mathrm{MAG}}+\frac{\zeta}{2} \mathcal{F}^{2}\left(g^{2}, \alpha\right) \widetilde{J}^{2}\right],
$$

where, for the moment, $\mathcal{F}\left(g^{2}, \alpha\right)$ is an arbitrary function of $\alpha$ of the form

$$
\mathcal{F}\left(g^{2}, \alpha\right)=1+f_{0}(\alpha) g^{2}+f_{1}(\alpha) g^{4}+O\left(g^{6}\right),
$$

and $\widetilde{J}$ is now the source. The generating functional becomes

$$
\exp (-i \widetilde{\mathcal{W}}(\widetilde{J}))=\int[D \phi] \exp i \widetilde{S}(\widetilde{J}) .
$$

Taking the functional derivative of $\widetilde{\mathcal{W}}(\widetilde{J})$ with respect to $\widetilde{J}$, we obtain

$$
\left.\frac{\delta \widetilde{\mathcal{W}}(\widetilde{J})}{\delta \widetilde{J}}\right|_{\widetilde{J}=0}=-\mathcal{F}\left(g^{2}, \alpha\right)\left\langle\mathcal{O}_{\mathrm{MAG}}\right\rangle
$$

Once more, we insert unity via

$$
1=\frac{1}{N} \int[D \widetilde{\sigma}] \exp \left[i \int d^{4} x\left(-\frac{1}{2 \zeta}\left(\frac{\widetilde{\sigma}}{g \mathcal{F}\left(g^{2}, \alpha\right)}-\mathcal{O}_{\mathrm{MAG}}-\zeta \widetilde{J} \mathcal{F}\left(g^{2}, \alpha\right)\right)^{2}\right)\right],
$$

to arrive at the following Lagrangian

$$
\widetilde{\mathcal{L}}\left(A_{\mu}, \widetilde{\sigma}\right)=-\frac{1}{4} F_{\mu \nu}^{a} F^{\mu \nu a}-\frac{1}{4} F_{\mu \nu}^{i} F^{\mu \nu i}+\mathcal{L}_{\mathrm{MAG}}+\mathcal{L}_{\mathrm{diag}}-\frac{\widetilde{\sigma}^{2}}{2 g^{2} \mathcal{F}^{2}\left(g^{2}, \alpha\right) \zeta}+\frac{1}{g^{2} \mathcal{F}\left(g^{2}, \alpha\right) \zeta} g \widetilde{\sigma} \mathcal{O}_{\mathrm{MAG}}-\frac{1}{2 \zeta}\left(\mathcal{O}_{\mathrm{MAG}}\right)^{2} .
$$

¿From the generating functional

$$
\begin{aligned}
\exp (-i \widetilde{\mathcal{W}}(\widetilde{J})) & =\int[D \phi] \exp i S_{\widetilde{\sigma}}(\widetilde{J}) \\
S_{\widetilde{\sigma}}(\widetilde{J}) & =\int d^{4} x\left(\mathcal{L}\left(A_{\mu}, \widetilde{\sigma}\right)+\widetilde{J} \frac{\widetilde{\sigma}}{g}\right) .
\end{aligned}
$$

it follows that

$$
\left.\frac{\delta \widetilde{\mathcal{W}}(\widetilde{J})}{\delta \widetilde{J}}\right|_{\widetilde{J}=0}=-\left\langle\frac{\widetilde{\sigma}}{g}\right\rangle \Rightarrow\langle\widetilde{\sigma}\rangle=g \mathcal{F}\left(g^{2}, \alpha\right)\left\langle\mathcal{O}_{\mathrm{MAG}}\right\rangle
$$


The renormalizability of the action (62) implies that the action (98) will be renormalizable too. Notice indeed that both actions are connected through the transformation

$$
\widetilde{J}=\frac{J}{\mathcal{F}\left(g^{2}, \alpha\right)} .
$$

The tree level off-diagonal masses are now provided by

$$
\begin{aligned}
& m_{\text {gluon }}^{\text {off-diag. }}=\sqrt{\frac{g \widetilde{\sigma}}{\zeta_{0}}}, \\
& m_{\text {ghost }}^{\text {off }- \text { diag. }}=\sqrt{\alpha \frac{g \widetilde{\sigma}}{\zeta_{0}}},
\end{aligned}
$$

while the vacuum configuration is determined by solving the gap equation

$$
\frac{d \widetilde{V}(\widetilde{\sigma})}{d \widetilde{\sigma}}=0
$$

with $\widetilde{V}(\widetilde{\sigma})$ the effective potential. Minimizing $\widetilde{V}(\widetilde{\sigma})$ will lead to a vacuum energy $E_{\text {vac }}(\alpha)$ which will depend on $\alpha$ and the hitherto undetermined functions $f_{i}(\alpha)$ [56]. We will determine those functions $f_{i}(\alpha)$ by requiring that $E_{\mathrm{vac}}(\alpha)$ is $\alpha$-independent. More precisely, one has

$$
\frac{d E_{\mathrm{vac}}}{d \alpha}=0 \Rightarrow \text { first order differential equations in } \alpha \text { for } f_{i}(\alpha)
$$

Of course, in order to be able to determine the $f_{i}(\alpha)$, we need an initial value for the vacuum energy $E_{\text {vac }}$. This corresponds to initial conditions for the $f_{i}(\alpha)$. In the case of the linear gauges, to fix the initial condition we employed the Landau gauge [33], a choice which would also be possible in case of the Curci-Ferrari gauges, since the Landau gauge belongs to these classes of gauges. This choice of the Landau gauge can be motivated by observing that the integrated operator $\int d^{4} x A_{\mu}^{A} A^{\mu A}$ has a gauge invariant meaning in the Landau gauge, due to the transversality condition $\partial_{\mu} A^{\mu A}=0$, namely

$$
(V T)^{-1} \min _{U \in S U(N)} \int d^{4} x\left[\left(A_{\mu}^{A}\right)^{U}\left(A^{\mu A}\right)^{U}\right]=\int d^{4} x\left(A_{\mu}^{A} A^{\mu A}\right) \text { in the Landau gauge },
$$

with the operator on the left hand side of eq.104) being gauge invariant. Moreover, the Landau gauge is also an all-order fixed point of the RGE for the gauge parameter in case of the linear and Curci-Ferrari gauges. At first glance, it could seem that it is not possible anymore to make use of the Landau gauge as initial condition in the case of the MAG, since the Landau gauge does not belong to the class of gauges we are currently considering. Fortunately, we shall be able to prove that we can use the Landau gauge as initial condition for the MAG too. This will be the content of the next section.

Before turning our attention to this task, it is worth noticing that, if one would work up to infinite order, the expressions (91) and (98) can be transformed exactly into those of (50), respectively (62) by means of eq.(100) and its associated transformation

$$
\widetilde{\sigma}=\mathcal{F}\left(g^{2}, \alpha\right) \sigma
$$

so that the effective potentials $\widetilde{V}(\widetilde{\sigma})$ and $V(\sigma)$ are exactly the same at infinite order, and as such will give rise to the same, gauge parameter independent, vacuum energy.

\section{INTERPOLATING BETWEEN THE MAG AND THE LANDAU GAUGE.}

In this section we shall introduce a generalized renormalizable gauge which interpolates between the MAG and the Landau gauge. This will provide a connection between these two gauges, allowing us to use the Landau gauge as initial condition. An example of such a generalized gauge, interpolating between the Landau and the Coulomb gauge was already presented in 42]. Moreover, we must realize that in the present case, we must also interpolate between the composite operator $\frac{1}{2} A_{\mu}^{A} A^{\mu A}$ of the Landau gauge and the gluon-ghost operator $\mathcal{O}_{\mathrm{MAG}}$ of the MAG. Although this seems to be a highly complicated assignment, there is an elegant way to treat it. 
Consider again the $S U(N)$ Yang-Mills action with the MAG gauge fixing (12). For the residual Abelian gauge freedom, we impose

$$
\begin{aligned}
S_{\mathrm{diag}}^{\prime} & =\int d^{4} x\left(b^{i} \partial_{\mu} A^{\mu i}+\bar{c}^{i} \partial^{2} c^{i}+\bar{c}^{i} \partial_{\mu}\left(g f^{i a b} A^{\mu a} c^{b}\right)+\kappa g f^{i a b} A_{\mu}^{a}\left(\partial_{\mu} c^{i}\right) \bar{c}^{b}+\kappa g^{2} f^{i a b} f^{i c d} \bar{c}^{a} c^{d} A_{\mu}^{b} A^{\mu c}\right. \\
& \left.-\kappa g f^{i a b} A_{\mu}^{i} A^{\mu a}\left(b^{b}-g f^{j b c} \bar{c}^{c} c^{j}\right)+\kappa g f^{i a b} A^{\mu i}\left(D_{\mu}^{a c} c^{c}\right) \bar{c}^{b}+\kappa g^{2} f^{a b i} f^{a c d} A_{\mu}^{i} A^{\mu c} c^{d} \bar{c}^{b}\right),
\end{aligned}
$$

where $\kappa$ is an additional gauge parameter. The gauge fixing (106) can be rewritten as a BRST exact expression

$$
S_{\text {diag }}^{\prime}=\int d^{4} x\left[(1-\kappa) s\left(\bar{c}^{i} \partial_{\mu} A^{\mu i}\right)+\kappa s \bar{s}\left(\frac{1}{2} A_{\mu}^{i} A^{\mu i}\right)\right] .
$$

Next, we will introduce the following generalized mass dimension two operator,

$$
\mathcal{O}=\frac{1}{2} A_{\mu}^{a} A^{\mu a}+\frac{\kappa}{2} A_{\mu}^{i} A^{\mu i}+\alpha \bar{c}^{a} c^{a}
$$

by means of

$$
\begin{aligned}
S_{\mathrm{LCO}}^{\prime} & =s \int d^{4} x\left(\lambda \mathcal{O}+\zeta \frac{\lambda J}{2}\right) \\
& =\int d^{4} x\left(J \mathcal{O}+\zeta \frac{J^{2}}{2}-\alpha \lambda b^{a} c^{a}+\lambda A^{\mu a} D_{\mu}^{a b} c^{b}+\alpha \lambda \bar{c}^{a}\left(g f^{a b i} c^{b} c^{i}+\frac{g}{2} f^{a b c} c^{b} c^{c}\right)\right. \\
& \left.-\kappa \lambda c^{i} \partial_{\mu} A^{\mu i}+\kappa g f^{i a b} \lambda A_{\mu}^{a} A^{\mu i} c^{b}\right),
\end{aligned}
$$

with $(J, \lambda)$ a BRST doublet of external sources,

$$
s \lambda=J, \quad s J=0 .
$$

As in the case of the gluon-ghost operator (18), the generalized operator of eq.(108) turns out to be BRST invariant on-shell, a property which can again be expressed in a functional way, see eq.(121).

Let us take a closer look at the action

$$
\Sigma^{\prime}=S_{\mathrm{YM}}+S_{\mathrm{MAG}}+S_{\mathrm{diag}}^{\prime}+S_{\mathrm{LCO}}^{\prime}+S_{\mathrm{ext}} .
$$

The external source part of the action, $S_{\text {ext }}$, is the same as given in eq.(16).

Also, it can be noticed that, for $\kappa \rightarrow 0$, the generalized local composite operator $\mathcal{O}$ of eq.(108) reduces to the composite operator $\mathcal{O}_{\mathrm{MAG}}$ of the MAG, while the diagonal gauge fixing (107) reduces to the Abelian Landau gauge (15). Said otherwise, for $\kappa \rightarrow 0$, the action $\Sigma^{\prime}$ of eq.(111) reduces to the one we are actually interested in and which we have discussed in the previous sections.

Another special case is $\kappa \rightarrow 1, \alpha \rightarrow 0$. Then the gauge fixing terms of $\Sigma^{\prime}$ are

$$
S_{\mathrm{MAG}}+S_{\mathrm{diag}}^{\prime}=\int d^{4} x s\left(-A_{\mu}^{A} \partial^{\mu} \bar{c}^{A}\right)=\int d^{4} x\left(\bar{c}^{A} \partial^{\mu} D_{\mu}^{A B} c^{B}+b^{A} \partial^{\mu} A_{\mu}^{A}\right),
$$

which is nothing else than the Landau gauge. At the same time, we also have

$$
\lim _{(\alpha, \kappa) \rightarrow(0,1)} \mathcal{O}=\frac{1}{2} A_{\mu}^{A} A^{\mu A},
$$

which is the pure gluon mass operator of the Landau gauge 21, 31].

¿From [31], we already know that the Landau gauge with the inclusion of the operator $A_{\mu}^{A} A^{\mu A}$ is renormalizable to all orders of perturbation theory. On the other hand, in section II, we have proven the renormalizability for $\kappa=0$. Before we continue our argument, let us first prove the renormalizability of $\Sigma^{\prime}$ for general $\alpha$ and $\kappa \neq 0$. The complete action $\Sigma^{\prime}$, as given in eq. (111), is BRST invariant

$$
s \Sigma^{\prime}=0
$$

and obeys the following identities 
- The Slavnov-Taylor identity, provided by

$$
\begin{aligned}
\mathcal{S}\left(\Sigma^{\prime}\right) & =\int d^{4} x\left(\frac{\delta \Sigma^{\prime}}{\delta \Omega^{\mu a}} \frac{\delta \Sigma^{\prime}}{\delta A_{\mu}^{a}}+\frac{\delta \Sigma^{\prime}}{\delta \Omega^{\mu i}} \frac{\delta \Sigma^{\prime}}{\delta A_{\mu}^{i}}+\frac{\delta \Sigma^{\prime}}{\delta L^{a}} \frac{\delta \Sigma^{\prime}}{\delta c^{a}}+\frac{\delta \Sigma^{\prime}}{\delta L^{i}} \frac{\delta \Sigma^{\prime}}{\delta c^{i}}\right. \\
& \left.+b^{a} \frac{\delta \Sigma^{\prime}}{\delta \bar{c}^{a}}+b^{i} \frac{\delta \Sigma^{\prime}}{\delta \bar{c}^{i}}+J \frac{\delta \Sigma^{\prime}}{\delta \lambda}\right)=0 .
\end{aligned}
$$

- The integrated diagonal ghost equation

$$
\mathcal{G}^{i} \Sigma^{\prime}=\Delta_{\mathrm{cl}}^{i}
$$

where

$$
\mathcal{G}^{i}=\int d^{4} x\left[\frac{\delta}{\delta c^{i}}+g f^{a b i} \bar{c}^{a} \frac{\delta}{\delta b^{b}}\right]
$$

and

$$
\Delta_{\mathrm{cl}}^{i}=\int d^{4} x\left[g f^{a b i} \Omega^{\mu a} A_{\mu}^{b}-g f^{a b i} L^{a} c^{b}+\kappa \lambda \partial_{\mu} A^{\mu i}\right],
$$

a classical breaking.

- The diagonal anti-ghost equation

$$
\frac{\delta \Sigma^{\prime}}{\delta \bar{c}^{i}}+\partial^{\mu} \frac{\delta \Sigma^{\prime}}{\delta \Omega^{\mu i}}=0
$$

and

$$
\frac{\delta \Sigma^{\prime}}{\delta b^{i}}=\partial_{\mu} A^{\mu i}
$$

- The integrated generalized $\lambda$-equation

$$
\int d^{4} x\left[\frac{\delta}{\delta \lambda}+c^{a} \frac{\delta}{\delta b^{a}}+\kappa c^{i} \frac{\delta}{\delta b^{i}}\right] \Sigma^{\prime}=0,
$$

expressing the on-shell BRST invariance of the operator $\mathcal{O}$ of eq. (108).

Also in this case, these Ward identities extend to the quantum level. Accordingly, the most general local counterterm $\Sigma^{\prime \text { count }}$ must obey the following constraints

$$
\begin{aligned}
\mathcal{B}_{\Sigma^{\prime}} \Sigma^{\text {count }} & =0, \\
\mathcal{G}^{i} \Sigma^{\prime \text { count }} & =0, \\
\frac{\delta}{\delta b^{i}} \Sigma^{\prime \text { count }} & =0, \\
{\left[\frac{\delta}{\delta \bar{c}^{i}}+\partial_{\mu} \frac{\delta}{\delta \Omega_{\mu}^{i}}\right] \Sigma^{\prime \text { count }} } & =0, \\
\int d^{4} x\left[\frac{\delta}{\delta \lambda}+c^{a} \frac{\delta}{\delta b^{a}}\right] \Sigma^{\prime \text { count }} & =0 .
\end{aligned}
$$

where $\mathcal{B}_{\Sigma^{\prime}}$ denotes the nilpotent, $\mathcal{B}_{\Sigma^{\prime}} \mathcal{B}_{\Sigma^{\prime}}=0$, linearized operator

$$
\begin{aligned}
\mathcal{B}_{\Sigma^{\prime}} & =\int d^{4} x\left(\frac{\delta \Sigma^{\prime}}{\delta \Omega^{a \mu}} \frac{\delta}{\delta A_{\mu}^{a}}+\frac{\delta \Sigma^{\prime}}{\delta A_{\mu}^{a}} \frac{\delta}{\delta \Omega^{a \mu}}+\frac{\delta \Sigma^{\prime}}{\delta \Omega^{\mu i}} \frac{\delta}{\delta A_{\mu}^{i}}+\frac{\delta \Sigma^{\prime}}{\delta A_{\mu}^{i}} \frac{\delta}{\delta \Omega^{\mu i}}+\frac{\delta \Sigma^{\prime}}{\delta L^{a}} \frac{\delta}{\delta c^{a}}\right. \\
& \left.+\frac{\delta \Sigma^{\prime}}{\delta c^{a}} \frac{\delta}{\delta L^{a}}+\frac{\delta \Sigma^{\prime}}{\delta L^{i}} \frac{\delta}{\delta c^{i}}+\frac{\delta \Sigma^{\prime}}{\delta c^{i}} \frac{\delta}{\delta L^{i}}+b^{a} \frac{\delta}{\delta \bar{c}^{a}}+b^{i} \frac{\delta}{\delta \bar{c}^{i}}+J \frac{\delta}{\delta \lambda}\right) .
\end{aligned}
$$


¿From general results on BRST cohomology [39], we know that the most general, local counterterm can be written as

$$
\Sigma^{\text {count }}=-\frac{a_{0}^{\prime}}{4} \int d^{4} x\left(F_{\mu \nu}^{a} F^{\mu \nu a}+F_{\mu \nu}^{i} F^{\mu \nu i}\right)+\mathcal{B}_{\Sigma} \Delta^{-1}
$$

where $\Delta^{-1}$ is an integrated local polynomial of ghost number -1 and dimension 4 , given by

$$
\begin{aligned}
\Delta^{-1} & =\int d^{4} x\left[a_{1}^{\prime} \Omega_{\mu}^{a} A^{\mu a}+a_{2}^{\prime} \Omega_{\mu}^{i} A^{\mu i}+a_{3}^{\prime} L^{a} c^{a}+a_{4}^{\prime} L^{i} c^{i}+a_{5}^{\prime}\left(\partial_{\mu} \bar{c}^{a}\right) A^{\mu a}+a_{5}^{\prime \prime} g f^{a b i} \bar{c}^{a} A_{\mu}^{i} A^{\mu b}\right. \\
& +a_{6}^{\prime}\left(\partial_{\mu} \bar{c}^{i}\right) A^{\mu i}+a_{7}^{\prime} g f^{a i c} \bar{c}^{a} \bar{c}^{i} c^{c}+\frac{a_{8}^{\prime}}{2} \alpha g f^{a b i} \bar{c}^{a} \bar{c}^{b} c^{i}+\frac{a_{9}^{\prime}}{2} \alpha g f^{a b c} \bar{c}^{a} \bar{c}^{b} c^{c}+\alpha a_{10}^{\prime} b^{a} \bar{c}^{a} \\
& \left.+a_{11}^{\prime} b^{i} \bar{c}^{i}+\frac{a_{12}^{\prime}}{2} \lambda A_{\mu}^{a} A^{\mu a}+\frac{a_{13}^{\prime}}{2} \kappa \lambda A_{\mu}^{i} A^{\mu i}+a_{14}^{\prime} \alpha \lambda \bar{c}^{a} c^{a}+a_{15}^{\prime} \alpha \lambda \bar{c}^{i} c^{i}+\frac{a_{16}^{\prime}}{2} \zeta \lambda J\right] .
\end{aligned}
$$

The constraints (122) lead to the relations

$$
\begin{aligned}
a_{7}^{\prime} & =a_{11}^{\prime}=a_{15}^{\prime}=0, \\
a_{6}^{\prime} & =a_{2}^{\prime}, \\
a_{13}^{\prime} & =a_{4}^{\prime}-a_{2}^{\prime}, \\
a_{8}^{\prime} & =-2 a_{10}^{\prime}, \\
a_{14}^{\prime} & =2 a_{10}^{\prime}+a_{3}^{\prime}, \\
a_{12}^{\prime} & =a_{3}^{\prime}-a_{5}^{\prime}, \\
a_{5}^{\prime \prime} & =a_{5}^{\prime}+\kappa\left(a_{13}^{\prime}-a_{3}^{\prime}\right), \\
a_{9}^{\prime} & =-a_{10}^{\prime}, \quad \text { and thus } a_{13}^{\prime}=0 . \\
a_{2}^{\prime} & =a_{4}^{\prime}=0 \quad
\end{aligned}
$$

Summarizing

$$
\begin{aligned}
\Delta^{-1} & =\int d^{4} x\left[a_{1}^{\prime} \Omega_{\mu}^{a} A^{\mu a}+a_{3}^{\prime} L^{a} c^{a}-a_{5}^{\prime} \bar{c}^{a} D_{\mu}^{a b} A^{\mu b}-\kappa a_{3}^{\prime} g f^{a b i} \bar{c}^{a} A_{\mu}^{i} A^{\mu b}-a_{10}^{\prime} \alpha g f^{a b i} \bar{c}^{a} \bar{c}^{b} c^{i}\right. \\
& \left.-\frac{a_{10}^{\prime}}{2} \alpha g f^{a b c} \bar{c}^{a} \bar{c}^{b} c^{c}+\alpha a_{10}^{\prime} b^{a} \bar{c}^{a}+a_{3}^{\prime} \lambda\left(\frac{1}{2} A_{\mu}^{a} A^{\mu a}+\alpha \bar{c}^{a} c^{a}\right)-\frac{a_{5}^{\prime}}{2} \lambda A_{\mu}^{a} A^{\mu a}+2 a_{10}^{\prime} \alpha \lambda \bar{c}^{a} c^{a}+\frac{a_{16}^{\prime}}{2} \zeta \lambda J\right]
\end{aligned}
$$

In comparison with the case of the MAG, we see that $\Sigma^{\prime \text { count }}$ also contains six free independent parameters, namely $a_{0}^{\prime}, a_{1}^{\prime}, a_{3}^{\prime}, a_{5}^{\prime}, a_{10}^{\prime}$ and $a_{13}^{\prime}$, despite the fact that the action $\Sigma^{\prime}$ contains the extra gauge parameter $\kappa$. These parameters can be reabsorbed by a suitable multiplicative renormalization of the gauge coupling constant $g$, of the gauge and LCO parameters $\alpha, \kappa, \zeta$, and of the fields $\phi=\left(A^{\mu a}, A_{\mu i}, c^{a}, \bar{c}^{a}, c^{i}, \bar{c}^{i}, b^{a}, b^{i}\right)$ and sources $\Phi=\left(\Omega^{\mu a}, \Omega^{\mu i}, L^{a}, L^{i}, \lambda\right.$, $J)$, according to

$$
\Sigma^{\prime}(g, \alpha, \kappa, \zeta, \phi, \Phi)+\eta \Sigma^{\prime \text { count }}=\Sigma^{\prime}\left(g_{0}, \alpha_{0}, \kappa_{0}, \zeta_{0}, \phi_{0}, \Phi_{0}\right)+O\left(\eta^{2}\right)
$$

where

$$
\begin{gathered}
g_{0}=Z_{g} g, \quad \alpha_{0}=Z_{\alpha} \alpha, \quad \zeta_{0}=Z_{\zeta} \zeta, \quad \kappa_{0}=Z_{c}^{-1} \widetilde{Z}_{A}^{-1 / 2} Z_{g}^{-1} \kappa, \\
A_{0}^{\mu a}=\widetilde{Z}_{A}^{1 / 2} A^{\mu a}, \\
c_{0}^{a}=\widetilde{Z}_{c}^{1 / 2} c^{a}, \quad \bar{c}_{0}^{a}=\widetilde{Z}_{c}^{1 / 2} \bar{c}^{a}, \\
c_{0}^{i}=Z_{c}^{1 / 2} c^{i}, \quad \bar{c}_{0}^{i}=Z_{c}^{-1 / 2} \bar{c}^{i}, \\
b_{0}^{a}=Z_{g} Z_{c}^{1 / 2} \widetilde{Z}_{c}^{1 / 2} b^{a}, \quad b_{0}^{i}=Z_{g} b^{i}, \\
\Omega_{0}^{\mu a}=\widetilde{Z}_{A}^{-1 / 2} Z_{g}^{-1} Z_{c}^{-1 / 2} \Omega^{\mu a}, \\
\Omega_{0}^{\mu i}=Z_{c}^{-1 / 2} \Omega^{i \mu}
\end{gathered}
$$




$$
\begin{gathered}
L_{0}^{a}=Z_{g}^{-1} \widetilde{Z}_{c}^{-1 / 2} Z_{c}^{-1 / 2} L^{a}, \quad L_{0}^{i}=Z_{g}^{-1} Z_{c}^{-1} L^{i}, \\
J_{0}=Z_{g}^{2} Z_{c} J, \quad \lambda_{0}=Z_{g} Z_{c}^{1 / 2} \lambda,
\end{gathered}
$$

with

$$
\begin{aligned}
Z_{g} & =1-\eta \frac{a_{0}^{\prime}}{2}, \\
Z_{\alpha} & =1+\eta\left(2 a_{5}^{\prime}+a_{0}^{\prime}-a_{8}^{\prime}\right), \\
Z_{\zeta} & =1+\eta\left(a_{16}^{\prime}+2 a_{0}^{\prime}+2 a_{5}^{\prime}-2 a_{3}^{\prime}\right), \\
\widetilde{Z}_{A}^{1 / 2} & =1+\eta\left(\frac{a_{0}^{\prime}}{2}+a_{1}^{\prime}\right), \\
\widetilde{Z}_{c}^{1 / 2} & =1-\frac{\eta}{2}\left(a_{5}^{\prime}+a_{3}^{\prime}\right), \\
Z_{c}^{1 / 2} & =1+\frac{\eta}{2}\left(a_{3}^{\prime}-a_{5}^{\prime}\right) .
\end{aligned}
$$

We see thus that the additional gauge parameter $\kappa$ does not renormalize in an independent way. Furthermore, from eq.(135), we notice that the relation (48) is generalized to the operator $\mathcal{O}$, i.e.

$$
\gamma_{\mathcal{O}}\left(g^{2}\right)=-2\left(\frac{\beta\left(g^{2}\right)}{2 g^{2}}-\gamma_{c^{i}}\left(g^{2}\right)\right)
$$

Summarizing, we have constructed a renormalizable gauge that is labelled by a couple of parameters $(\alpha, \kappa)$. It allows us to introduce a generalized composite operator $\mathcal{O}$, given by eq.(108), which embodies the local operator $A_{\mu}^{A} A^{\mu A}$ of the Landau gauge as well as the operator $\mathcal{O}_{\mathrm{MAG}}$ of the MAG. To construct the effective potential, one sets all sources equal to zero, except $J$, and introduces unity to remove the $J^{2}$ terms. A completely analogous argument as the one given in section III allows to conclude that the minimum value of $V(\sigma)$, thus $E_{\mathrm{vac}}$, will be independent of $\alpha$ and $\kappa$, essentially because the derivative with respect to $\alpha$ as well as with respect to $\kappa$ is BRST exact, up to terms in the source $J$. This independence of $\alpha$ and $\kappa$ is again only assured at infinite order in perturbation theory, so we can generalize the construction, proposed in section III, by making the function $\mathcal{F}$ of eq.921) also dependent on $\kappa$. The foregoing analysis is sufficient to make sure that we can use the Landau gauge result for $E_{\mathrm{vac}}$ as the initial condition for the vacuum energy of the MAG. Moreover, we are now even in the position to answer the question about the existence of a fixed point of the RGE for the gauge parameter $\alpha$, which was necessary to certify that no arbitrary constants would enter the results for $E_{\mathrm{vac}}$. We already mentioned that the Landau gauge, i.e. the case $(\alpha, \kappa)=(0,1)$, is a renormalizable model [31], i.e. the Landau gauge is stable against radiative corrections. This can be reexpressed by saying that $(\alpha, \kappa)=(0,1)$ is a fixed point of the RGE for the gauge parameters, and this to all orders of perturbation theory.

\section{NUMERICAL RESULTS FOR $S U(2)$.}

After a quite lengthy formal construction of the LCO formalism in the case of the MAG, we are now ready to present explicit results. In this paper, we will restrict ourselves to the evaluation of the one-loop effective potential in the case of $S U(2)$. As renormalization scheme, we adopt the modified minimal substraction scheme $(\overline{\mathrm{MS}})$. Let us give here, for further use, the values of the one-loop anomalous dimensions of the relevant fields and couplings in the case of $S U(2)$. In our conventions, one has [43, 44, 45]

$$
\begin{aligned}
& \gamma_{c^{i}}\left(g^{2}\right)=(-3-\alpha) \frac{g^{2}}{16 \pi^{2}}+O\left(g^{4}\right), \\
& \gamma_{\alpha}\left(g^{2}\right)=\left(-2 \alpha+\frac{8}{3}-\frac{6}{\alpha}\right) \frac{g^{2}}{16 \pi^{2}}+O\left(g^{4}\right),
\end{aligned}
$$

while

$$
\beta\left(g^{2}\right)=-\varepsilon g^{2}-2\left(\frac{22}{3} \frac{g^{4}}{16 \pi^{2}}\right)+O\left(g^{6}\right)
$$


and exploiting the relation (48)

$$
\gamma_{O_{\mathrm{MAG}}}\left(g^{2}\right)=\left(\frac{26}{3}-2 \alpha\right) \frac{g^{2}}{16 \pi^{2}}+O\left(g^{4}\right)
$$

a result consistent with that of [4].

The reader will notice that we have given only the 1-loop values of the anomalous dimensions, despite the fact that we have announced that one needs $(n+1)$-loop knowledge of the RGE functions to determine the $n$-loop potential. As we shall see soon, the introduction of the function $\mathcal{F}\left(g^{2}, \alpha\right)$ and the use of the Landau gauge as initial condition allow us to determine the 1-loop results we are interested in, from the 1-loop RGE functions only.

Let us first determine the counterterm $\delta \zeta$. For the generating functional $\mathcal{W}(J)$, we find at 1-loop [57]

$$
\mathcal{W}(J)=\int d^{d} x\left(-(\zeta+\delta \zeta) \frac{J^{2}}{2}\right)+i \ln \operatorname{det}\left[\delta^{a b}\left(\partial^{2}+\alpha J\right)\right]-\frac{i}{2} \ln \operatorname{det}\left[\delta^{a b}\left(\left(\partial^{2}+J\right) g_{\mu \nu}-\left(1-\frac{1}{\alpha}\right) \partial_{\mu} \partial_{\nu}\right)\right]
$$

and employing

$$
\ln \operatorname{det}\left[\delta^{a b}\left(\left(\partial^{2}+J\right) g_{\mu \nu}-\left(1-\frac{1}{\alpha}\right) \partial_{\mu} \partial_{\nu}\right)\right]=\delta^{a a}\left[(d-1) \operatorname{tr} \ln \left(\partial^{2}+J\right)+\operatorname{tr} \ln \left(\partial^{2}+\alpha J\right)\right]
$$

with

$$
\delta^{a a}=N(N-1)=2 \text { for } N=2,
$$

one can calculate the divergent part of eq.(142),

$$
\mathcal{W}(J)=\int d^{4} x\left[-\delta \zeta \frac{J^{2}}{2}-\frac{3}{16 \pi^{2}} J^{2} \frac{1}{\varepsilon}-\frac{1}{16 \pi^{2}} \alpha^{2} J^{2} \frac{1}{\varepsilon}+\frac{1}{8 \pi^{2}} \alpha^{2} J^{2} \frac{1}{\varepsilon}\right] .
$$

Consequently,

$$
\delta \zeta=\frac{1}{8 \pi^{2}}\left(\alpha^{2}-3\right) \frac{1}{\varepsilon}+O\left(g^{2}\right) .
$$

Next, we can compute the RGE function $\delta\left(g^{2}, \alpha\right)$ from eq.(55), obtaining

$$
\delta\left(g^{2}, \alpha\right)=\frac{\alpha^{2}-3}{8 \pi^{2}}+O\left(g^{2}\right)
$$

Having determined this, we are ready to calculate $\zeta_{0}$. The differential equation (67) is solved by

$$
\zeta_{0}(\alpha)=\alpha+\left(9-4 \alpha+3 \alpha^{2}\right) C_{0}
$$

with $C_{0}$ an integration constant. As already explained in the previous sections, we can consistently put $C_{0}=0$. Here, we have written it explicitly to illustrate that, if $\alpha$ would coincide with the 1-loop fixed point of the RGE for the gauge parameter, the part proportional to $C_{0}$ in eq.(148) would drop. Indeed, the equations $9-4 \alpha+3 \alpha^{2}=0$ and $-2 \alpha+\frac{8}{3}-\frac{6}{\alpha}=0$, stemming from eq.(139), are the same. Moreover, we also notice that this equation has only complex valued solutions. Therefore, it is even more important to have made the connection between the MAG and the Landau gauge by embedding them in a bigger class of gauges, since then we have the fixed point, even at all orders. In what follows, it is understood that $\zeta_{0}=\alpha$.

We now have all the ingredients to construct the 1-loop effective potential $\widetilde{V}_{1}(\widetilde{\sigma})$,

$$
\begin{aligned}
\widetilde{V}_{1}(\tilde{\sigma})= & \frac{\tilde{\sigma}^{2}}{2 \zeta_{0}}\left(1-\left(2 f_{0}+\frac{\zeta_{1}}{\zeta_{0}}\right) g^{2}\right)+i \ln \operatorname{det}\left[\delta^{a b}\left(\partial^{2}+\alpha \frac{g \tilde{\sigma}}{\zeta_{0}}\right)\right] \\
& -\frac{i}{2} \ln \operatorname{det}\left[\delta^{a b}\left(\left(\partial^{2}+\frac{g \tilde{\sigma}}{\zeta_{0}}\right) g_{\mu \nu}-\left(1-\frac{1}{\alpha}\right) \partial_{\mu} \partial_{\nu}\right)\right]
\end{aligned}
$$

or, after renormalization

$$
\widetilde{V}_{1}(\widetilde{\sigma})=\frac{\tilde{\sigma}^{2}}{2 \zeta_{0}}\left(1-\left(2 f_{0}+\frac{\zeta_{1}}{\zeta_{0}}\right) g^{2}\right)+\frac{3}{32 \pi^{2}} \frac{g^{2} \widetilde{\sigma}^{2}}{\zeta_{0}^{2}}\left(\ln \frac{g \widetilde{\sigma}}{\zeta_{0} \bar{\mu}^{2}}-\frac{5}{6}\right)-\frac{1}{32 \pi^{2}} \frac{g^{2} \alpha^{2} \widetilde{\sigma}^{2}}{\zeta_{0}^{2}}\left(\ln \frac{g \alpha \widetilde{\sigma}}{\zeta_{0} \bar{\mu}^{2}}-\frac{3}{2}\right) .
$$


We did not explicitly write the divergences and counterterms in eq.151), since by construction we know that the formalism is renormalizable, so they would have cancelled amongst each other. This can be checked explicitly by using the unity of (59) with counterterms included. It can also be checked explicitly that $\widetilde{V}_{1}(\widetilde{\sigma})$ obeys the renormalization group

$$
\mu \frac{d}{d \mu} \widetilde{V}_{1}(\widetilde{\sigma})=0+\text { terms of higher order },
$$

by using the RGE functions (138)-(141) and the fact that the anomalous dimension of $\widetilde{\sigma}$ is given by

$$
\gamma_{\tilde{\sigma}}\left(g^{2}\right)=\frac{\beta\left(g^{2}\right)}{2 g^{2}}+\gamma_{\mathcal{O}_{\mathrm{MAG}}}\left(g^{2}\right)+\mu \frac{\partial \ln \mathcal{F}\left(g^{2}, \alpha\right)}{\partial \mu},
$$

which is immediately verifiable from eq. (99).

We now search for the vacuum configuration by minimizing $\widetilde{V}_{1}(\widetilde{\sigma})$ with respect to $\widetilde{\sigma}$. We will put $\bar{\mu}^{2}=\frac{g \widetilde{\sigma}}{\zeta_{0}}$ to exclude possibly large logarithms, and find two solutions of the gap equation

$$
\begin{aligned}
& \left.\frac{d \widetilde{V}_{1}}{d \sigma}\right|_{\bar{\mu}^{2}=\frac{g \widetilde{\sigma}}{\zeta_{0}}}=0 \\
\Leftrightarrow & \frac{\widetilde{\sigma}}{\zeta_{0}}\left(1-\left(2 f_{0}+\frac{\zeta_{1}}{\zeta_{0}}\right) g^{2}\right)+\frac{3}{16 \pi^{2}} \frac{g^{2} \widetilde{\sigma}}{\zeta_{0}^{2}}\left(-\frac{5}{6}\right)+\frac{3}{32 \pi^{2}} \frac{g^{2} \widetilde{\sigma}}{\zeta_{0}^{2}}-\frac{1}{16 \pi^{2}} \frac{g^{2} \alpha^{2} \widetilde{\sigma}}{\zeta_{0}^{2}}\left(\ln \alpha-\frac{3}{2}\right)-\frac{1}{32 \pi^{2}} \frac{g^{2} \alpha^{2} \widetilde{\sigma}}{\zeta_{0}^{2}}=0,
\end{aligned}
$$

namely

$$
\begin{aligned}
& \widetilde{\sigma}=0, \\
& \left.y \equiv \frac{g^{2} N}{16 \pi^{2}}\right|_{N=2}=\frac{2 \zeta_{0}}{16 \pi^{2}\left(2 f_{0} \zeta_{0}+\zeta_{1}\right)+\alpha^{2} \ln \alpha-\alpha^{2}+1} .
\end{aligned}
$$

The quantity $y$ is the relevant expansion parameter, and should be sufficiently small to have a sensible expansion. The value for $\langle\widetilde{\sigma}\rangle$ corresponding to eq. (155) can be extracted from the 1-loop coupling constant

$$
g^{2}(\bar{\mu})=\frac{1}{\beta_{0} \ln \frac{\bar{\mu}^{2}}{\Lambda \frac{2}{\mathrm{MS}}}} .
$$

The first solution (154) corresponds to the usual, perturbative vacuum ( $\left.E_{\mathrm{vac}}=0\right)$, while eq. (155) gives rise to a dynamically favoured vacuum with energy

$$
\begin{aligned}
E_{\mathrm{vac}} & =-\frac{1}{64 \pi^{2}}\left(3-\alpha^{2}\right)\left(m_{\mathrm{gluon}}^{\text {off }- \text { diag }}\right)^{4}, \\
m_{\text {gluon }}^{\text {off }- \text { diag }} & =e^{\frac{3}{22 y}} \Lambda_{\overline{\mathrm{MS}}} .
\end{aligned}
$$

Eq.(157) is obtained upon substitution of eq.(153) into eq.(150). From eq.(157), we notice that at the 1-loop approximation, $\alpha^{2} \leq 3$ must be fulfilled in order to have $E_{\mathrm{vac}} \leq 0$. In principle, the unknown function $f_{0}(\alpha)$ can be determined by solving the differential equation

$$
\begin{aligned}
\frac{d E_{\mathrm{vac}}}{d \alpha}=0 & \Leftrightarrow 2 \alpha\left(m_{\mathrm{gluon}}^{\text {off }- \text { diag }}\right)^{4}+4\left(\alpha^{2}-3\right)\left(m_{\mathrm{gluon}}^{\text {off-diag }}\right)^{3} \frac{d m_{\mathrm{gluon}}^{\text {off-diag }}}{d \alpha}=0 \\
& \Leftrightarrow \alpha+\frac{3-\alpha^{2}}{y^{2}}\left(\frac{\partial y}{\partial \alpha}+\frac{\partial y}{\partial \zeta_{0}} \frac{\partial \zeta_{0}}{\partial \alpha}+\frac{\partial y}{\partial \zeta_{1}} \frac{\partial \zeta_{1}}{\partial \alpha}+\frac{\partial y}{\partial f_{0}} \frac{\partial f_{0}}{\partial \alpha}\right)=0
\end{aligned}
$$

with initial condition $E_{\mathrm{vac}}(\alpha)=E_{\mathrm{vac}}^{\text {Landau }}$. However, to solve eq. (159) knowledge of $\zeta_{1}$ is needed. Since we are not interested in $f_{0}(\alpha)$ itself, but rather in the value of the vacuum energy $E_{\mathrm{vac}}$, the off-diagonal mass $m_{\text {gluon }}^{\text {off }}$-diag and the expansion parameter $y$, there is a more direct way to proceed, without having to solve the eq.(159). Let us first give the Landau gauge value for $E_{\mathrm{vac}}$ in the case $N=2$, which can be easily obtained from [21, 47],

$$
E_{\mathrm{vac}}^{\mathrm{Landau}}=-\frac{9}{128 \pi^{2}} e^{\frac{17}{6}} \Lambda_{\mathrm{MS}} .
$$




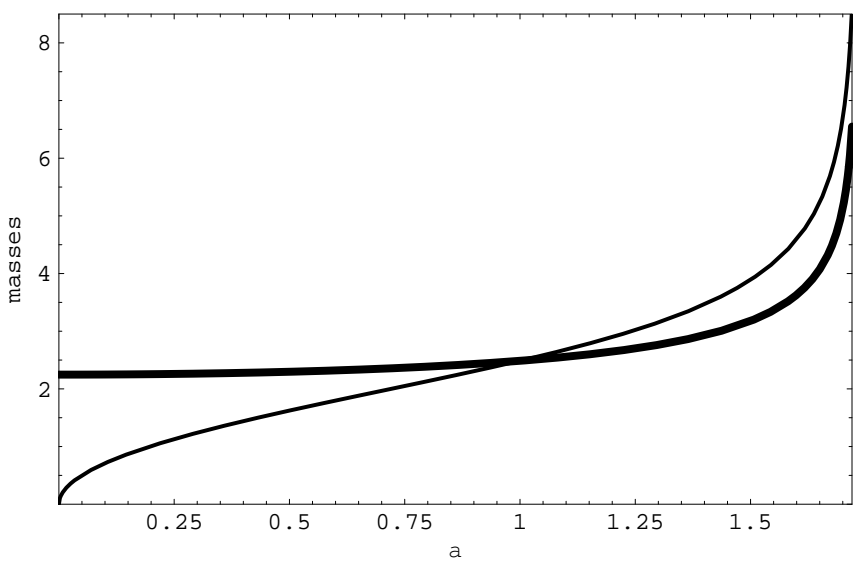

FIG. 1: The off-diagonal gluon (fat line) and ghost mass (thin line) in function of $\alpha$. Masses are in units of $\Lambda_{\overline{\mathrm{MS}}}$.

Since the construction is such that $E_{\mathrm{vac}}(\alpha)=E_{\mathrm{vac}}^{\text {Landau }}$, we can equally well solve

$$
-\frac{9}{128 \pi^{2}} e^{\frac{17}{6}} \Lambda_{\overline{\mathrm{MS}}}^{4}=-\frac{1}{64 \pi^{2}}\left(3-\alpha^{2}\right)\left(m_{\text {gluon }}^{\text {off }- \text { diag }}\right)^{4}
$$

which gives the lowest order mass

$$
m_{\text {gluon }}^{\text {off-diag }}=\left(\frac{9}{2} \frac{e^{\frac{17}{6}}}{3-\alpha^{2}}\right)^{\frac{1}{4}} \Lambda_{\overline{\mathrm{MS}}}
$$

and hence

$$
m_{\text {ghost }}^{\text {off }- \text { diag }}=\sqrt{\alpha}\left(\frac{9}{2} \frac{e^{\frac{17}{6}}}{3-\alpha^{2}}\right)^{\frac{1}{4}} \Lambda_{\overline{\mathrm{MS}}},
$$

The result (162) can be used to determine $y$. From eq.(158) one easily finds

$$
y=\frac{36}{187+66 \ln \frac{9}{2\left(3-\alpha^{2}\right)}} .
$$

We see thus that, for the information we are currently interested in, we do not need explicit knowledge of $\zeta_{1}$ and $f_{0}$. We want to remark that, if $\zeta_{1}$ were known, the value for $y$ obtained in eq. (164) can be used to determine $f_{0}$ from eq.(155). This is a nice feature, since the possibly difficult differential equation (159) never needs to be solved in this fashion. In Fig. 1, we have plotted the off-diagonal gluon mass (162) and ghost mass (163) for $0 \leq \alpha \leq \sqrt{3}$. We notice that the masses grow to $\infty$ for increasing $\alpha$, while the expansion parameter $y$ drops to zero, as it is clear from Fig. 2. The relative smallness of $y$ means that our perturbative analysis should give qualitatively meaningful results. Before we come to the conclusions, let us consider the limit $\alpha \rightarrow 0$, corresponding to the "real" MAG $D_{\mu}^{a b} A^{\mu b}=0$. One finds

$$
\begin{aligned}
m_{\text {gluon }}^{\text {off-diag }} & =\left(\frac{3}{2} e^{\frac{17}{6}}\right)^{\frac{1}{4}} \Lambda_{\overline{\mathrm{MS}}} \approx 2.25 \Lambda_{\overline{\mathrm{MS}}}, \\
y & =\frac{36}{187+66 \ln \frac{3}{2}} \approx 0.168
\end{aligned}
$$

\section{DISCUSSION AND CONCLUSION.}

The aim of this paper was to give analytic evidence, as expressed by eq. 165), of the dynamical mass generation for off-diagonal gluons in Yang-Mills theory quantized in the maximal Abelian gauge. This mass can be seen as support 


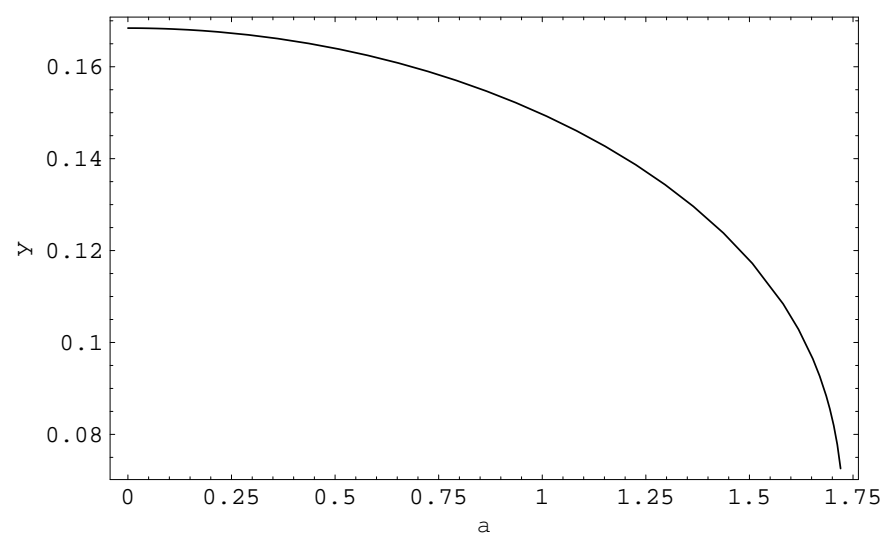

FIG. 2: The expansion parameter $y$ as a function of $\alpha$.

for the Abelian dominance [3, 4, [5] in that gauge. This result is in qualitative agreement with the lattice version of the MAG, were such a mass was also reported [10, 11]. The off-diagonal lattice gluon propagator could be fitted by $\frac{1}{p^{2}+m^{2}}$, which is in correspondence with the tree level propagator we find. We have been able to prove the existence of the off-diagonal mass by investigating the condensation of a mass dimension two operator, namely $\left(\frac{1}{2} A_{\mu}^{a} A^{\mu a}+\alpha \bar{c}^{a} c^{a}\right)$. It was shown how a meaningful, renormalizable effective potential for this local composite operator can be constructed. By evaluating this potential explicitly at 1-loop order in the case of $S U(2)$, the formation of the condensate is favoured since it lowers the vacuum energy. The latter does not depend on the choice of the gauge parameter $\alpha$, at least if one would work to infinite order in perturbation theory. We have explained in short the problem at finite order and discussed a way to overcome it. Moreover, we have been able to interpolate between the Landau gauge and the MAG by unifying them in a larger class of renormalizable gauges. This observation was used to prove that the vacuum energy of Yang-Mills theory in the MAG due to its mass dimension two condensate should be the same as the vacuum energy of Yang-Mills theory in the Landau gauge with the much explored condensate $\left\langle A_{\mu}^{A} A^{\mu A}\right\rangle$. It is worth noticing that all the gauges, where a dimension two condensate provides a dynamical gluon mass parameter, such as the Landau gauge 21], the Curci-Ferrari gauges [20], the linear gauges [33] and the MAG, can be connected to each other, either directly (e.g. Landau-MAG) or via the Landau gauge (e.g. MAG and linear gauges). This also implies that, if $\left\langle A_{\mu}^{A} A^{\mu A}\right\rangle \neq 0$ in the Landau gauge, the analogous condensates in the other gauges cannot vanish either. Then the question arises if this correspondence between different gauges could be stretched further to for instance the Coulomb gauge, where the possibility of a condensate $\left\langle A_{i}^{A} A^{i A}\right\rangle$ was already advocated some time ago in [48]. However, it is worth remarking that this might be a more complicated task, since the Coulomb gauge is not a covariant gauge fixing, and as such its analysis within the algebraic remormalization framework [30] is not straightforward.

Needless to say, the present work is far from being complete. First of all, an explicit calculation at two loop order and for general gauge group $S U(N)$ would be interesting. We also limited our computations to the tree level order. In principle, one should evaluate the off-diagonal gluon polarization in order to get further information on the structure of the propagator. A first step in this direction was taken in the case of the Landau gauge in [46]. It is unknown what will happen at higher orders in the MAG, but it is likely that the external momentum $Q^{2}$ will enter through loop corrections and influence the possible position of a pole in the propagator. The ghost condensation, that was first investigated in 12, 13] as a possible mechanism behind the off-diagonal mass, and later on was shown to be tachyonic [14, 15], could enter this polarization too. This would require a more complete treatment of the ghost condensation in the MAG, along the lines of [49], where these condensates were considered in more detail in the case of the Curci-Ferrari and Landau gauge. Another issue which deserves attention is the behaviour of the diagonal gluon. In [1], it was found that the diagonal gluon propagator also contain a mass parameters, with $m_{\text {gluon }}^{\text {diag }} \approx \frac{1}{2} m_{\text {gluon }}^{\text {off }- \text { diag }}$, while in [10] the diagonal gluon was reported to behave like a light or massless particle. For completeness, we remind that these lattice simulations were both performed in the case of $S U(2)$. We want to remark that a condensation of the composite diagonal operator $A_{\mu}^{i} A^{\mu i}$ cannot occur within our approach, since this is forbidden by the diagonal local $U(1)^{N-1}$ Ward identity (30). In principle, one could add an extra source term like $\frac{1}{2} \rho A_{\mu}^{i} A^{\mu i}$, but it does not seem possible to prove the renormalizability of this operator in the MAG. This might be consistent with the result of [1], since the diagonal gluon propagator could not be fitted with a Yukawa propagator $\frac{1}{p^{2}+m^{2}}$, in contrast with the off-diagonal gluon propagator which could be fitted with $\frac{1}{p^{2}+m^{2}}$. This could mean that the diagonal mass parameter is of a different nature compared to the off-diagonal one. A possible speculation is that it might have to do with 
Gribov copies, since a fit $\frac{p^{2}}{p^{4}+m^{4}}$ did work for the diagonal propagator [1].

Our analysis of the MAG condensate was also restricted to the purely perturbative level. One could imagine calculating in a certain non-trivial background. The vacuum energy calculated in one gauge should still be the same as the one calculated in the other gauge. In this context, and keeping in mind that monopole condensation is an essential ingredient of the dual superconductor picture, it might be worth noticing that the role of $\left\langle A_{u t}^{A} A^{\mu A}\right\rangle$ as an order parameter for monopole condensation was investigated in the Landau gauge by the authors of [26], based on a similar observation in compact QED 25]. We note that an off-diagonal gluon mass can serve as a starting point to derive low energy (dual) Abelian models for Yang-Mills theories, see for example [50, 51, 52].

Let us conclude with a few considerations on the issues of the degrees of freedom and of the unitarity when the gluons attain a dynamical mass, as a consequence of a nonvanishing dimension two condensate $\left\langle\mathcal{O}_{\mathrm{MAG}}\right\rangle=\left\langle\frac{1}{2} A_{\mu}^{a} A^{\mu a}+\alpha \bar{c}^{a} c^{a}\right\rangle$. One possible way to look at the degrees of freedom associated to a given field is through its propagator. From the pole of the propagator one gets information about the mass of the field, while from its residue one learns about polarization states. However, the propagation of the field has to occur in some vacuum. In other words, the kind of vacuum in which the field propagates has to be supplemented. In our case, this task is achieved by the LCO Lagrangian, eq.(60), i.e.

$$
\mathcal{L}\left(A_{\mu}, \sigma\right)=-\frac{1}{4} F_{\mu \nu}^{a} F^{\mu \nu a}-\frac{1}{4} F_{\mu \nu}^{i} F^{\mu \nu i}+\mathcal{L}_{\mathrm{MAG}}+\mathcal{L}_{\mathrm{diag}}-\frac{\sigma^{2}}{2 g^{2} \zeta}+\frac{1}{g^{2} \zeta} g \sigma \mathcal{O}_{\mathrm{MAG}}-\frac{1}{2 \zeta}\left(\mathcal{O}_{\mathrm{MAG}}\right)^{2},
$$

which allows one to take into account the effects related to having a nontrivial vacuum corresponding to the nonvanishing dimension two condensate $\left\langle\mathcal{O}_{\mathrm{MAG}}\right\rangle$, as expressed by the identity

$$
\langle\sigma\rangle=g\left\langle\mathcal{O}_{\mathrm{MAG}}\right\rangle
$$

That this is the preferred vacuum follows from the observation that the vacuum energy is lowered by the condensate $\left\langle\mathcal{O}_{\mathrm{MAG}}\right\rangle$. Expanding thus around $\langle\sigma\rangle \neq 0$, a dynamical tree level mass $m_{\text {gluon }}^{\text {off-diag. }}$ for the off-diagonal gluons is generated in the gauge fixed Lagrangian (166), namely

$$
m_{\text {gluon }}^{\text {off }- \text { diag. }}=\sqrt{\frac{g\langle\sigma\rangle}{\zeta_{0}}} .
$$

Therefore, in the condensed vacuum, $\langle\sigma\rangle \neq 0$, the Lagrangian (166) accounts for off-diagonal massive gluons. However, we emphasize that this dynamical mass parameter occurs as the result of a particular condensate. It is not a free parameter of the gauge fixed theory, its value being determined by a gap equation. Concerning now the unitarity of the resulting theory, it should be remarked that, due to confinement, gluons and quarks are only to be called physical at a very high energy scale $Q^{2}$, where they behave almost freely and asymptotic states can be related to them, thanks to asymptotic freedom. At very high energies, our dynamically massive action might be unitary: a renormalization group improvement could induce quantum corrections such that the mass parameter runs to zero for $Q^{2} \rightarrow \infty$. Otherwise said, the corrections induced by this dynamical mass on the scattering amplitudes are expected to become less and less important as the energy of the process increases, so that the amplitudes of the massless case are in fact recovered. Such a scenario would be analogous to the behaviour of the dynamical mass parameter discussed by Cornwall in [53]. For very high $Q^{2}$, one does indeed expect that perturbative Yang-Mills theory with massless gluons, having two physical degrees of freedom, describes the physical spectrum and that non-perturbative corrections are absent.

To decide if our resulting theory is unitary at smaller $Q^{2}$, one should know how to take into account the effects of confinement, which now cannot be neglected. This would amount to knowing how to construct out of our Lagrangian (166) the low energy spectrum of the theory, which is believed to be given by colorless bound states of gluons and quarks as, for instance, mesons, baryons and glueballs. This task is far beyond our capabilities. At intermediate $Q^{2}$, what we can state is that this dynamical mass parametrizes the behaviour of the Greens function of the gluon. As a result of quantum effects, i.e. the condensation of the mass dimension two operator, a pole appears in the off-diagonal gluon propagator at the tree level. Including higher order effects will alter the propagators behaviour as well as the location of the pole at physical $Q^{2}$ (i.e. $Q^{2}<0$ ). In the case of the Landau gauge, higher order calculations showed that the condensate remains stable, and hence a nonzero mass parameter will remain, see [21, 47]. This mass parameter will describe the behaviour of the Greens function at Euclidean $Q^{2}$. The presence of a mass parameter does however not necessarily entail the presence of a pole in the propagator at physical $Q^{2}$. Using lattice simulations of the Euclidean propagator, a mass parameter is found also by fitting at Euclidean $Q^{2}>0$, but no pole and related massive particle is implied. Analogously, one should not conclude from our calculations that the gluon is a massive, physical particle and that unitarity is violated. 


\section{Acknowledgments.}

The Conselho Nacional de Desenvolvimento Científico e Tecnológico (CNPq-Brazil), the SR2-UERJ and the Coordenação de Aperfeiçoamento de Pessoal de Nível Superior (CAPES) are gratefully acknowledged for financial support. D. Dudal would like to thank the Theoretical Physics Department of the UERJ for the kind hospitality where a part of this work was completed, while R. F. Sobreiro wants to acknowledge the warm hospitality at the Department of Mathematical Physics and Astronomy where another part of this work was completed.

[1] Y. Nambu, Phys. Rev. D10 (1974) 4262;

G. 't Hooft, High Energy Physics EPS Int. Conference, Palermo 1975, ed. A. Zichichi;

S. Mandelstam, Phys. Rept. 23 (1976) 245.

[2] G. 't Hooft, Nucl. Phys. B 190 (1981) 455.

[3] Z. F. Ezawa and A. Iwazaki, Phys. Rev. D 25 (1982) 2681.

[4] T. Suzuki and I. Yotsuyanagi, Phys. Rev. D 42 (1990) 4257.

[5] S. Hioki, S. Kitahara, S. Kiura, Y. Matsubara, O. Miyamura, S. Ohno and T. Suzuki, Phys. Lett. B 272 (1991) 326 [Erratum-ibid. B 281 (1992) 416].

[6] A. S. Kronfeld, G. Schierholz and U. J. Wiese, Nucl. Phys. B 293 (1987) 461.

[7] A. S. Kronfeld, M. L. Laursen, G. Schierholz and U. J. Wiese, Phys. Lett. B 198 (1987) 516.

[8] H. Min, T. Lee and P. Y. Pac, Phys. Rev. D 32 (1985) 440.

[9] A. R. Fazio, V. E. R. Lemes, M. S. Sarandy and S. P. Sorella, Phys. Rev. D 64 (2001) 085003.

[10] K. Amemiya and H. Suganuma, Phys. Rev. D 60 (1999) 114509.

[11] V. G. Bornyakov, M. N. Chernodub, F. V. Gubarev, S. M. Morozov and M. I. Polikarpov, Phys. Lett. B 559 (2003) 214.

[12] M. Schaden, hep-th/9909011

[13] K. I. Kondo and T. Shinohara, Phys. Lett. B 491 (2000) 263.

[14] D. Dudal and H. Verschelde, J. Phys. A 36 (2003) 8507.

[15] H. Sawayanagi, Phys. Rev. D 67 (2003) 045002.

[16] K. I. Kondo, Phys. Lett. B 514 (2001) 335.

[17] G. Curci and R. Ferrari, Nuovo Cim. A 32 (1976) 151.

[18] G. Curci and R. Ferrari, Phys. Lett. B 63 (1976) 91.

[19] D. Dudal, H. Verschelde, V. E. R. Lemes, M. S. Sarandy, R. F. Sobreiro, S. P. Sorella, M. Picariello, J.A. Gracey, Phys. Lett. B 569 (2003) 57.

[20] D. Dudal, H. Verschelde, V. E. R. Lemes, M. S. Sarandy, S. P. Sorella and M. Picariello, Annals Phys. 308 (2003) 62.

[21] H. Verschelde, K. Knecht, K. Van Acoleyen and M. Vanderkelen, Phys. Lett. B 516 (2001) 307.

[22] K. Langfeld, H. Reinhardt and J. Gattnar, Nucl. Phys. B 621 (2002) 131.

[23] A. C. Aguilar and A. A. Natale, hep-ph/0405024

[24] A. C. Aguilar and A. A. Natale, JHEP 0408 (2004) 057.

[25] F. V. Gubarev, L. Stodolsky and V. I. Zakharov, Phys. Rev. Lett. 86 (2001) 2220.

[26] F. V. Gubarev and V. I. Zakharov, Phys. Lett. B 501 (2001).

[27] P. Boucaud, A. Le Yaouanc, J. P. Leroy, J. Micheli, O. Pène and J. Rodriguez-Quintero, Phys. Lett. B 493 (2000) 315.

[28] P. Boucaud, A. Le Yaouanc, J. P. Leroy, J. Micheli, O. Pène and J. Rodriguez-Quintero, Phys. Rev. D 63 (2001) 114003.

[29] P. Boucaud, J. P. Leroy, A. Le Yaouanc, J. Micheli, O. Pène, F. De Soto, A. Donini, H. Moutarde and J. Rodríguez-Quintero Phys. Rev. D 66 (2002) 034504.

[30] O. Piguet and S.P. Sorella, Algebraic Renormalization, Lect. Notes Phys. M28 (1995) 1.

[31] D. Dudal, H. Verschelde and S. P. Sorella, Phys. Lett. B 555 (2003) 126.

[32] D. Dudal, H. Verschelde, V. E. R. Lemes, M. S. Sarandy, R. F. Sobreiro, S. P. Sorella and J. A. Gracey, Phys. Lett. B 574 (2003) 325 .

[33] D. Dudal, H. Verschelde, J. A. Gracey, V. E. R. Lemes, M. S. Sarandy, R. F. Sobreiro and S. P. Sorella, JHEP 0401 (2004) 044 .

[34] G. Parisi and R. Petronzio, Phys. Lett. B 94 (1980) 51.

[35] J. H. Field, Phys. Rev. D 66 (2002) 013013.

[36] F. Giacosa, T. Gutsche and A. Faessler, hep-ph/0408085

[37] K. I. Kondo, Phys. Rev. D 57 (1998) 7467.

[38] K. I. Kondo, T. Murakami, T. Shinohara and T. Imai, Phys. Rev. D 65 (2002) 085034.

[39] G. Barnich, F. Brandt and M. Henneaux, Phys. Rept. 338, 439 (2000).

[40] H. Verschelde, Phys. Lett. B 351 (1995) 242.

[41] K. Knecht and H. Verschelde, Phys. Rev. D 64 (2001) 085006.

[42] L. Baulieu and D. Zwanziger, Nucl. Phys. B 548 (1999) 527.

[43] T. Shinohara, T. Imai and K. I. Kondo, Int. J. Mod. Phys. A 18 (2003) 5733.

[44] U. Ellwanger and N. Wschebor, Int. J. Mod. Phys. A 18 (2003) 1595. 
[45] K. I. Kondo, hep-th/0303251

[46] R. E. Browne and J. A. Gracey, Phys. Lett. B 597 (2004) 368.

[47] R. E. Browne and J. A. Gracey, JHEP 0311 (2003) 029.

[48] J. Greensite and M. B. Halpern, Nucl. Phys. B 271 (1986) 379.

[49] D. Dudal, H. Verschelde, V. E. R. Lemes, M. S. Sarandy, S. P. Sorella, M. Picariello, A. Vicini and J. A. Gracey, JHEP 0306 (2003) 003.

[50] K. I. Kondo, hep-th/0009152

[51] S. Deguchi and Y. Kokubo, Mod. Phys. Lett. A 18 (2003) 2051.

[52] F. Freire, Phys. Lett. B 526 (2002) 405.

[53] J. M. Cornwall, Phys. Rev. D 26 (1982) 1453.

[54] The index $a$ runs only over the $N(N-1)$ off-diagonal generators.

[55] See section V.

[56] At first order, $E_{\mathrm{vac}}$ will depend on $f_{0}(\alpha)$, at second order on $f_{0}(\alpha)$ and $f_{1}(\alpha)$, etc.

[57] We will do the transformation of $\mathcal{W}(J)$ to $\mathcal{W}(\widetilde{J})$ only at the end. 